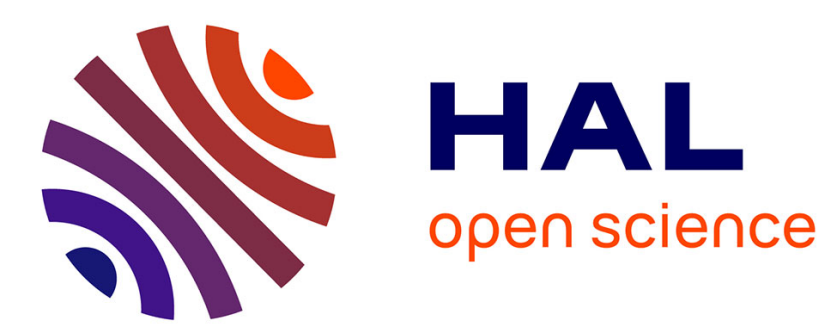

\title{
Thermal evolution of a metal drop falling in a less dense, more viscous fluid
}

B. Qaddah, Julien Monteux, M. Le Bars

\section{To cite this version:}

B. Qaddah, Julien Monteux, M. Le Bars. Thermal evolution of a metal drop falling in a less dense, more viscous fluid. Physical Review Fluids, 2020, 5, pp.053801. 10.1103/PhysRevFluids.5.053801 . hal-02563299

\section{HAL Id: hal-02563299 \\ https://hal.science/hal-02563299}

Submitted on 5 May 2020

HAL is a multi-disciplinary open access archive for the deposit and dissemination of scientific research documents, whether they are published or not. The documents may come from teaching and research institutions in France or abroad, or from public or private research centers.
L'archive ouverte pluridisciplinaire HAL, est destinée au dépôt et à la diffusion de documents scientifiques de niveau recherche, publiés ou non, émanant des établissements d'enseignement et de recherche français ou étrangers, des laboratoires publics ou privés. 


\author{
B. Qaddah ${ }^{\mathrm{a}, \mathrm{b}}$, J. Monteux ${ }^{\mathrm{b}}$, M. Le Bars ${ }^{\mathrm{a}}$ \\ ${ }^{a}$ CNRS, Aix Marseille Univ, Centrale Marseille, IRPHE, Marseille, France \\ ${ }^{b}$ Université Clermont Auvergne, CNRS, IRD, OPGC, Laboratoire Magmas et Volcans, \\ F-63000 Clermont-Ferrand, France \\ Corresponding author: qaddah@irphe.univ-mrs.fr
}

\begin{abstract}
9 The initial state of terrestrial planets was partly determined, during accretion,
Abstract

by the fall of metal drops in a liquid magma ocean. Here, we perform systematic numerical simulations in 2D cylindrical axisymmetric geometry of these falling dynamics and associated heat exchanges at the scale of one single drop, for various initial sizes and ambient viscosities. We explore Reynolds number in the range [0.05-48], viscosity ratios in the range [50 - 4000], Weber number in the range $[0.04-5]$ and Peclet number in the range $[70-850]$. We show that heat exchanges between the two phases occurs predominantly at the front section of the drop. Our systematic, parametric study exhibits shows that the thermal boundary layer thickness, the depth and time for equilibration, the Nusselt number, and the magma ocean volume affected by thermal echanges, all scale as power laws of the Peclet number. Because of drop distortions, these scaling laws deviate from the classical balances considering only heat diffusion through a laminar thermal boundary layer. Finally, when considering a temperaturedependent viscosity of the ambient fluid, we show that a low viscosity layer surrounds the drop, which influences the thermal evolution of non-deformable, low Reynolds number drops only, and decreases the breakup distance for some limited breakup modes.
\end{abstract}

\section{Thermal evolution of a metal drop falling in a less dense, more viscous fluid}


Keywords: Core formation, drop dynamics, thermal exchanges, two-phase flow, numerical modeling.

\section{Introduction}

Core formation of terrestrial planets is a complex process contemporaneous with planetary accretion (1; 2). Its fluid dynamics and thermodynamics have been addressed in numerous studies (e.g. 3, 4, 5, 6, 7, 8). During the last stages of e.g. Earth accretion, giant impacts likely occurred between the proto-Earth and up to Mars-sized differentiated bodies (9). The kinetic energy released during such collisions $(10$; 11), the radioactive heating caused by the disintegration of short-lived radio-elements (12), and the heat dissipation resulting from the conversion of potential energy during core formation and core/mantle separation (13), melt part or all of the Earth mantle (10). Following each impact, the iron core of the impactor thus spreaded and sank into a deep magma ocean. There, the metal further fragmented into drops and diapirs of different sizes, ranging from millimeter drops up to maybe kilometers diapirs, before assembling with the Earth proto-core ((5, 7, 14; 15)). Thermochemical exchanges occured between the fragmented metal drops and the liquid magma ocean during their sinking, determining the initial thermal and chemical state of the planet $((16 ; 17 ; 18,5))$. Past studies have provided many scenarios to characterize and quantify the thermochemical exchanges. (16) and (19) modelled the diffusive equilibration through a laminar thermal boundary layer of respectively, a cloud of uniform drops and a large diapir of iron. (18) further evaluated the influence of drop deformations in (16) scenario. (20) solved the fully coupled dynamical and thermal/chemical equations, but for a fixed spherical geometry only. (5) used experiments where a large volume of immiscible fluid falls into a less dense ambient to show that the smallest scale of turbulence - rather than diffusion 
through a laminar boundary layer - leads to rapid thermo-chemical equilibration, even before fragmentation. (8) confirmed this conclusion in their analog model, measuring the global cooling of a large volume of hot Galinstan after its fall through a deep tank of viscous oil. Yet a systematic temporal description of heat exchanges at the scale of one falling, freely evolving drop, is still missing.

Importantly, the magma ocean viscosity highly depends on its evolving temperature and pressure (21). Therefore, the viscosity ratio between the magma ocean and iron drops can vary by several orders of magnitude as a function of depth, of time after impact, etc. Following and extending an abundant literature in different contexts (e.g. 22, 23, 24; 25, 26), analog experiments by (7, 8) and numerical simulations at the scale of one metal drop by (27) showed that the viscosity contrast indeed plays an important role in iron drops shape, velocity and fragmentation. (27) predicted that thermo-chemical exchanges should increase with drop deformation and oscillations; but they did not explicitly solve for the fully coupled dynamical and thermal equations. This is the purpose of the present paper. Open questions include: How and where do heat exchanges occur? Do the drop deformation/oscillations indeed favor heat exchanges? What are the characteristics time and depth needed to reach equilibration between the two phases? And, what is the influence of a temperature dependent viscosity of the magma?

The paper is organised as follows. Section 2 introduces the physical and numerical models, with the governing equations, the non-dimensional parameters, and the numerical method. Section 3 presents in detail a reference case, describing its mechanical and thermal behavior, average temperature evolution, heat transfer at the drop interface, and the magma ocean volume heated during the drop sinking. In Section 4, we present the main numerical results from our systematic parametric study, and derive generic scaling laws for the 
above detailed parameters. Section 5 then focuses on changes induced by a temperature-dependent viscosity in the magma ocean. Conclusions and future works are outlined in final section 6 .

\section{Physical and numerical models}

\subsection{Governing equations}

We consider an initially spherical, liquid metal drop of radius $R$, falling in an initially motionless, less dense and more viscous surrounding fluid (i.e. a magma ocean) under the action of gravity. The initial temperature of the

liquid drop and of the magma ocean strongly depends on the growth history of the protoplanet before the impact and on its initial heating caused by short lived elements (13). Here, we consider that the liquid metal drop is hotter than the magma ocean, with uniform initial temperatures in both phases. Both phases behave as Newtonian, incompressible, and immiscible fluids with uniform surface tension, and constant density and viscosity within each fluid at first. Later on in section 5, we also consider a temperature dependent magma ocean viscosity. The dynamical and thermal evolution of the falling drop and ambient liquid is governed by the Navier-Stokes and heat transfer equations, describing - The mass conservation:

$$
\nabla \cdot u=0
$$

${ }_{99} \quad$ with $\boldsymbol{u}$ the fluid velocity vector $\left(\mathrm{m} \cdot \mathrm{s}^{-1}\right)$.

- The momentum conservation:

$$
\rho\left(\frac{\partial \boldsymbol{u}}{\partial t}+\boldsymbol{u} \cdot \nabla \boldsymbol{u}\right)=\nabla \cdot\left[-P \boldsymbol{I}+\mu\left(\nabla \boldsymbol{u}+(\nabla \boldsymbol{u})^{T}\right)\right]+\rho \boldsymbol{g}+\boldsymbol{F}_{s t}
$$


with $\rho$ the fluid density $\left(\mathrm{kg} \cdot \mathrm{m}^{-3}\right), \mu$ the fluid dynamic viscosity (Pa.s), $t$ the time (s), $P$ the fluid pressure $(\mathrm{Pa}), \boldsymbol{g}$ the gravitational acceleration $\left(\mathrm{m} . \mathrm{s}^{-2}\right), \boldsymbol{F}_{s t}$ the surface tension force $\left(\mathrm{N} . \mathrm{m}^{-3}\right)$ and $\boldsymbol{I}$ the identity matrix.

- The heat conservation:

$$
\rho C_{p}\left[\frac{\partial T}{\partial t}+\boldsymbol{u} \cdot \nabla T\right]+\nabla \cdot[-k \nabla T]=0
$$

with $C_{p}$ the heat capacity at constant pressure $\left(\mathrm{J} \cdot \mathrm{kg}^{-1} \cdot \mathrm{K}^{-1}\right), T$ the fluid temperature $(\mathrm{K}), k$ the fluid thermal conductivity $\left(\mathrm{W} \cdot \mathrm{m}^{-1} \cdot \mathrm{K}^{-1}\right)$. No heat source is considered in our model. We ignore the effect of viscous dissipation in this work as we study droplets on small scales of millimetres to centimetres. The absence of viscous dissipation for these studied drops does not affect the thermal evolution in the system.

To monitor the interface between the falling drop and the magma ocean, we use the Level Set method, an Eulerian and implicit method frequently used in multiphase flow problems (e.g. 28). The Level Set function $\phi$ equals to 1 in the metal drop and 0 in the ambient liquid, and rapidly changes through the interface, whose position is determined by the isocontour $\phi=0.5$. The transport and reinitialization of the Level Set function $\phi$ are governed by:

$$
\frac{\partial \phi}{\partial t}+\boldsymbol{u} \cdot \nabla \phi=\gamma \nabla \cdot\left[\epsilon \nabla \phi-\phi(1-\phi) \frac{\nabla \phi}{|\nabla \phi|}\right]
$$

with $\gamma(\mathrm{m} / \mathrm{s})$ and $\epsilon(\mathrm{m})$ the reinitialization parameters. $\gamma$ determines the reinitialization amount: a suitable value for $\gamma$ is the maximum velocity magnitude experienced in the model. $\epsilon$ determines the layer thickness around the interface, and is equal to half the size of the characteristic mesh in the region explored by the interface. The density and dynamical viscosity are evaluated using the level set function: 
123

$$
\rho=\rho_{m}+\left(\rho_{d}-\rho_{m}\right) \phi
$$

$$
\mu=\mu_{m}+\left(\mu_{d}-\mu_{m}\right) \phi
$$

where subscripts "m" and "d" stand for the magma ocean and the liquid metal drop, respectively. The surface tension force is determined by :

$$
\boldsymbol{F}_{s t}=\nabla \cdot \boldsymbol{T}=\nabla \cdot\left(\sigma\left[\boldsymbol{I}+\left(-\boldsymbol{n} \boldsymbol{n}^{T}\right)\right] \delta\right)
$$

with $\sigma(\mathrm{N} / \mathrm{m})$ the surface tension coefficient, $I$ the identity matrix, $\boldsymbol{n}$ the interface normal unit vector, and $\delta$ the Dirac delta function, nonzero only at the fluid interface. The interface normal unit vector is calculated as

$$
\boldsymbol{n}=\frac{\nabla \phi}{|\nabla \phi|} .
$$

The level set parameter $\phi$ is also used to approximate the delta function by a smooth function (29) defined by

$$
\delta=6|\phi(1-\phi)||\nabla \phi| .
$$

Note that in this work we do not calculate the chemical exchanges between the two phases. Instead, the main difference between the heat and mass transfer equations, if the thermal and chemical sources are neglected, is the partition coefficient between the two phases (30). When the partition coefficient is equal to 1 , we can use the results of this study in geochemical models of planet building (31).

\subsection{Physical and non-dimensional parameters}

The main parameters that characterize the dynamical and thermal evolution of a falling drop in a more viscous medium are the viscosity, density, thermal 
conductivity, heat capacity and initial temperature of the two fluids, the initial drop size, the gravity, and the surface tension between the two phases. In the geophysical problem of interest (i.e. core formation), the magma ocean viscosity and the metal drop initial radius vary over a wide range of values, while the other parameters are roughly constant (even if rigorously, the thermal conductivity and heat capacity of a magma ocean moderately depend on its composition (e.g. 32 ; 33), and the thermal conductivity and heat capacity of metal drops moderately depend on temperature and pressure (e.g. 34; 35)). Hence in this study, we vary these two parameters $R$ and $\mu_{m}$, in the accessible, relevant ranges 4-25 $\mathrm{mm}$ and $0.25-20 \mathrm{~Pa} . \mathrm{s}$ respectively, while we keep all the other parameters fixed at their representative geophysical values (see Table 1).

Table 1: Symbol definitions and values of the physical and non-dimensional parameters used in this study.

\begin{tabular}{lcc}
\hline & Symbol & Value or range \\
\hline \hline Magma ocean density & $\rho_{m}$ & $3500 \mathrm{~kg} / \mathrm{m}^{3}$ \\
\hline Metal drop density & $\rho_{d}$ & $7500 \mathrm{~kg} / \mathrm{m}^{3}$ \\
\hline Metal drop viscosity & $\mu_{d}$ & $0.005 \mathrm{~Pa} . \mathrm{s}$ \\
\hline Magma ocean viscosity & $\mu_{m}$ & $0.25-20 \mathrm{~Pa} . \mathrm{s}$ \\
\hline Initial drop radius & $R$ & $4-25 \mathrm{~mm}$ \\
\hline Surface tension coefficient & $\sigma$ & $1 \mathrm{~N} / \mathrm{m}$ \\
\hline Magma ocean heat capacity & $C p_{m}$ & $667 \mathrm{J.kg}^{-1} . \mathrm{K}^{-1}$ \\
\hline Metal heat capacity & $C p_{d}$ & $800 \mathrm{~J} . \mathrm{kg}^{-1} . \mathrm{K}^{-1}$ \\
\hline Magma ocean conductivity & $k_{m}$ & $10 \mathrm{~W} . \mathrm{m}^{-1} . \mathrm{K}^{-1}$ \\
\hline Metal conductivity & $k_{d}$ & $100 \mathrm{~W} . \mathrm{m}^{-1} . \mathrm{K}^{-1}$ \\
\hline Viscosity ratio & $R_{\mu}$ & $50-4000$ \\
\hline Density ratio & $R_{\rho}$ & 2.14 \\
\hline Reynolds number & $R e$ & $0.05-48$ \\
\hline Weber number & $W e$ & $0.04-5$ \\
\hline Peclet number & $P e$ & $70-850$ \\
\hline Nusselt number & $N u$ & $1-6$ \\
\hline
\end{tabular}

In our simulations, the drop falls from rest, accelerates until reaching a constant terminal velocity, possibly with small oscillations around it, and thermally 
exchanges heat with the ambient liquid. We pursue continue our simulations until the drop reaches a stable dynamical regime and its temperature contrast with the ambient reaches less than $20 \%$ of its initial value. During the fall, we monitor the average terminal velocity $V$ and the average temperature $T$ of the drop (minus the initial ambient temperature). The dynamical and thermal evolution of each drop is then characterised by the following output dimensionless numbers:

- the Reynolds number ( $\left.R e=\frac{\rho_{m} V R}{\mu_{m}}\right)$ is the ratio of inertial to viscous forces. Three different regimes are possible: the Stokes regime corresponds to $R e<1$ where the viscous effects dominate; the Intermediate regime corresponds to $R e=1-500$ where both viscous and inertial forces are important; and the Newtonian regime corresponds to $R e>500$ where the inertial forces are dominant. Here, the Reynolds number ranges from 0.05 to 48 , hence our drops are in the Stokes to Intermediate regime.

- the Weber number $\left(W e=\frac{\rho_{m} V^{2} R}{\sigma}\right)$ compares the inertial and surface tension forces. It governs the deformation, breakup and terminal shape of a drop (see e.g. 36, 27). When $W e<O(1)$, the drop remains spherical without any change of its morphology, while increasing Weber number leads to stronger and stronger deformation, then to fragmentation above a threshold which increases with the viscosity ratio, starting from $\sim 3$ for viscosity ratio $\leq 1$ (see e.g. 37,27 ). Here, the Weber number ranges from 0.04 to 5 , considering stable, potentially deformable drops only.

- the Peclet number $\left(P e=\frac{\rho_{m} C p_{m} V R}{k_{m}}\right)$ compares the rate of heat advection to diffusion at the drop scale. Here, the Peclet number ranges from 70 to 850 , so heat transfers are is strongly affected by advection.

- the Nusselt number $\left(N u=\frac{R \overline{\nabla T} \cdot \boldsymbol{n}}{\left(\overline{T_{i n t}}-T_{m}\right)}\right)$ compares the measured, averaged 
heat transfer at the drop interface to a purely conductive case, with $\overline{T_{i n t}}$ the mean temperature at the interface and $T_{m}$ the magma ocean temperature far from the drop. Here, the Nusselt number ranges from 1 to 6 , hence confirming the important role of advection in heat transfers.

All relevant parameter values are given in Table 1.

\subsection{Numerical model}

We solve Eqs. (1- 4) using axisymmetric simulations with the COMSOL Multiphysics software, based on the finite element method. The details of our 17 runs for this study are listed in Table 2, Each run represents 2 to 4 weeks computation time on a bi-processor, eight-core, $3.2-3.6 \mathrm{GHz}$ workstation. The axisymmetric geometry assumption is validated by (38) for a Weber number up to 120 . For the dynamics, we use open conditions at the top and bottom boundaries and no-slip conditions at the lateral boundary. For the temperature, we consider no flux conditions at all boundaries. The computational domain must be large enough to allow for convergence without any wall effects. Here, we chose an axisymmetric cylinder of size $(r \times z)=(12 R \times 200 R)$, which is sufficiently large to reach a statistically steady motion (see our previous study (27)) and to follow equilibration up to a $80 \%$ decrease of the initial temperature anomaly.

To capture precisely the dynamical and thermal evolution of the drop, a fine mesh is required. For that, we use an adaptive mesh with a high resolution in the drop vicinity. As shown in figure 1, we divide our domain into several regions where the cell sizes vary between $h=0.015 R$ to $h=1.5 R$. As detailed in (27), the simulation is programmed to stop when the drop reaches the bottom of the finest mesh region: the whole mesh pattern is then translated and the simulation is restarted on this new grid (see Figure 1 Right). 
Table 2: Dimensional and non-dimensional parameters for all performed simulations used in this study. $P e_{h}$ is the grid Peclet number (see section 2.3.

\begin{tabular}{lcccccccc}
\hline Simulation & $R(\mathrm{~mm})$ & $\mu_{m}(P a . s)$ & $R_{\mu}$ & $R e$ & $W e$ & $P e$ & $P e_{h}$ & $\Delta T(K)$ \\
\hline$\# 1$ & 8 & 20 & 4000 & 0.05 & 0.04 & 70 & 0.43 & 100 \\
\hline$\# 2$ & 8 & 10 & 2000 & 0.2 & 0.15 & 137.26 & 0.84 & 100 \\
\hline$\# 3$ & 8 & 5 & 1000 & 0.76 & 0.52 & 254 & 1.55 & 100 \\
\hline$\# 3 \mathrm{a}\left(T\right.$ dependent $\left.\mu_{m}\right)$ & 8 & 5 & 1000 & 0.81 & 0.59 & 271 & 1.65 & 100 \\
\hline$\# 3 \mathrm{~b}\left(T\right.$ dependent $\left.\mu_{m}\right)$ & 8 & 5 & 1000 & 0.98 & 0.85 & 327 & 2 & 1000 \\
\hline$\# 4$ & 8 & 1 & 200 & 9.5 & 3.23 & 635 & 3.89 & 100 \\
\hline$\# 5$ & 8 & 0.5 & 100 & 21.8 & 4.26 & 728.36 & 4.46 & 100 \\
\hline$\# 5 \mathrm{a}\left(T\right.$ dependent $\left.\mu_{m}\right)$ & 8 & 0.5 & 100 & 22.4 & 4.48 & 747.6 & 4.57 & 100 \\
\hline$\# 5 \mathrm{~b}\left(T\right.$ dependent $\left.\mu_{m}\right)$ & 8 & 0.5 & 100 & 23.52 & 4.94 & 785 & 4.8 & 1000 \\
\hline$\# 6$ & 8 & 0.25 & 50 & 47.6 & 5 & 793.73 & 4.86 & 100 \\
\hline$\# 6 \mathrm{a}\left(T\right.$ dependent $\left.\mu_{m}\right)$ & 8 & 0.25 & 50 & 47.6 & 5 & 793.73 & 4.86 & 100 \\
\hline$\# 6 \mathrm{~b}\left(T\right.$ dependent $\left.\mu_{m}\right)$ & 8 & 0.25 & 50 & 47.6 & 5 & 793.73 & 4.86 & 1000 \\
\hline$\# 7$ & 4 & 1 & 200 & 2.2 & 0.34 & 145.67 & 0.89 & 100 \\
\hline$\# 8$ & 6 & 1 & 200 & 5.67 & 1.53 & 378.19 & 2.32 & 100 \\
\hline$\# 9$ & 10 & 1 & 200 & 12.7 & 4.61 & 847.42 & 5.2 & 100 \\
\hline$\# 10$ & 25 & 1 & 200 & 45 & 23.2 & 3008 & 18.45 & 100 \\
\hline$\# 10 \mathrm{a}\left(T\right.$ dependent $\left.\mu_{m}\right)$ & 25 & 1 & 200 & 41.13 & 19.5 & 2745 & 16.8 & 1000 \\
\hline
\end{tabular}

Mesh convergence has been checked. In (27), we showed that a mesh size $h=0.025 R$ (or smaller) allows to capture the falling drop dynamics. Here, we further performed two tests for the resolution of thermal transfers. In the first one, we compare the numerical and analytical heat transfers by thermal diffusion from a motionless spherical drop. The analytical solution for the radial temperature profile from the drop center to a given distance (r) is given by (39)

$$
T=0.5 T_{0}\left[\operatorname{erf} \frac{R+r}{2 \sqrt{D t}}+\operatorname{erf} \frac{R-r}{2 \sqrt{D t}}\right]-\frac{T_{0}}{r} \sqrt{\frac{D t}{\pi}}\left[e^{\frac{-(R-r)^{2}}{4 D t}}-e^{\frac{-(R+r)^{2}}{4 D t}}\right]
$$

with $T$ the temperature anomaly ( $T_{0}$ its initial value) and $D$ the thermal diffusivity $\left(m^{2} \cdot \mathrm{s}^{-1}\right)$. Figure 2(Left) shows the excellent agreement of our numerical results. 
For the second test, we calculated, for different minimum grid sizes $h=$ $0.05 R, 0.035 R, 0.025 R, 0.015 R, 0.01 R$, the normalized average drop temperature as a function of normalized time for our reference case \#4 in Table 2 (see details in next section). Figure 2 (Right) shows a reasonable convergence of the numerical results from $h=0.015 R$, with a relative maximum error $\simeq 3.5 \%$, while the drop mass during the course of this simulation does not change by more than $0.4 \%$ from its initial mass. Therefore, we confirm that our mesh $h=0.015 R$ captures correctly the thermal evolution of the metal drop.

Finally, the grid Peclet number $P e_{h}=\frac{V h}{D_{i n t}}$, with $D_{i n t}=\frac{D_{d}+D_{m}}{2}$ the mean thermal diffusivity, is an appropriate parameter in the convection-diffusion equation to determine whether the heat transfer in the system corresponds to numerical artifacts or not. (40) found very accurate solutions for grid Peclet number up to 10 . As shown in Table 2, our runs in this study remain below this criterion for stability, except for run \#10 which we hence do not consider for heat transfer studies in the following sections.

\section{Reference case}

In this section, we present our reference case, from which, in the next section, we then change the drop size keeping the viscosity ratio constant, and the magma ocean viscosity keeping the drop size constant. This reference case corresponds to simulation \#4 in Table 2 the drop initial radius is $8 \mathrm{~mm}$, the magma ocean viscosity is 1 Pa.s, the metal viscosity is 0.005 Pa.s (viscosity contrast 200), and the initial temperature difference between the metal drop and the magma ocean is $100 \mathrm{~K}$.

The drop motion from rest and its thermal evolution are shown in Figure 3 . The spherical drop accelerates due to gravity and rapidly deforms into a spherical cap. Then, surface tension equilibrates inertia at the drop interface and 
prevents any further change in morphology. Higher temperatures are concentrated at the front of the drop, while the thermal wake behind the drop expands as a function of time. To characterize this dynamics, we define and compute the following quantities.

\subsection{Average fallingdrop velocity}

During its fall, the drop mean velocity varies with time depending on the drop morphology. In our simulations, we compute it as:

$$
V(t)=\frac{\int_{v} U(r, z, t)[\phi(r, z, t) \geq 0.5] \mathrm{d} v}{\int_{v}[\phi(r, z, t) \geq 0.5] \mathrm{d} v}
$$

with $U(r, z, t)$ the local velocity magnitude $(\mathrm{m} / \mathrm{s}), \mathrm{d} v=2 \pi r \mathrm{~d} r \mathrm{~d} z$ accounting for axisymmetric cylindrical geometry, and $[\phi(r, z, t) \geq 0.5]$ the boolean operator allowing to only capture the iron drop volume. Note that we use in this study the mean magnitude velocity of drop which is very close to the average vertical velocity (change of less than $3 \%$ ). We normalise $V(t)$ by the free fall Newton velocity $U_{N}=\sqrt{\frac{\Delta \rho g R}{\rho_{m}}}$ and time by the diffusion time $t_{d i f}=\frac{R^{2} \rho_{m} C p_{m}}{k_{m}}$. Figure 4 shows the result for our reference case. The drop rapidly accelerates from rest up to $t^{*}=0.02$, then several small oscillations occur before reaching its asymptotic terminal fall velocity, which we use to compute the output dimensionless numbers.

\subsection{Average temperature evolution of the metal drop}

As shown in Figure 3, the liquid drop exchanges heat with the liquid magma ocean and loses its heat as a function of time and depth. We determine the mean average temperature anomaly of the drop compared to the magma ocean as:

$$
T(t)=\frac{\int_{v} T(r, z, t)[\phi \geq 0.5] \mathrm{d} v}{\int_{v}[\phi \geq 0.5] \mathrm{d} v}
$$


We normalize $T(t)$ by its initial value, giving $T^{*}(t)$. Its evolution for the reference case as a function of time or as a function of depth of its center of mass (normalized by the initial radius $R$ ) is shown in Figure 5 . After a rapid adjustment of the initial temperature jump at the interface, $T^{*}$ decreases exponentially towards equilibrium. We determine the best exponential fits according to

$$
T^{*}=e^{\left(-t / t_{c}\right)} \quad \text { and } \quad T^{*}=C_{z} e^{\left(-z / l_{c}\right)}
$$

where $C_{z}$ is a constant (see Figure 5 in red color). In geophysical science, the characteristic time $\left(t_{c}\right)$ and length $\left(l_{c}\right)$ for equilibration are very important parameters (8), used to set the degree of equilibration between iron and silicate of magma ocean in planet building models (31). Note that chemical equilibrium is more difficult to reach than thermal equilibrium because of its low diffusivity and is at least as important to planet formation as thermal equilibration, especially for small metal volumes. Therefore, in the case of a partition coefficient between the two phases equal to 1 , the results of this work can be used in geochemical models of planet building.

\subsection{Volume of heated magma during the drop sinking}

The magma ocean temperature increases during the fall of the drop, especially in its wake. It is important in geophysics to quantify how much of the magma is thermally affected by the formation of the iron core: this would for instance affect the initial structure and heat budget of the Earth's mantle. In our simulations, we calculate the volume of magma ocean affected by thermal exchange as:

$$
V o=\int_{v}\left[T>\left(T_{m}+T_{C}\right)\right][\phi \leq 0.5] \mathrm{d} v
$$

with $T_{C}$ a chosen temperature anomaly $(K)$ and $[\phi \leq 0.5]$ the boolean operator allowing to only capture the magma ocean volume. An example of the captured 
volume of heated magma ocean is shown in Figure 6 (Left). We performed tests with several $T_{C}$ corresponding to $0.1,1$ and $10 \mathrm{~K}$. Results of the volume normalized by the initial drop volume as a function of the drop depth are shown in Figure 6 (Right). The three curves corresponding to different equilibrium degrees are parallel, until a depth equals to $50 \mathrm{R}$ where $T_{C}=10 \mathrm{~K}$ is strongly affected by thermal diffusion in the magma ocean. The same behavior would of course take place at longer time/depth for the other $T_{C}$. In the following, for our parametric study, we compare heated volumes considering $T_{C}=1 \mathrm{~K}$ and at three different depths: 10, 20 and $30 \mathrm{R}$.

\subsection{Heat transfer at the drop interface}

To evaluate the Nusselt number, we compute the mean temperature anomaly and the mean temperature gradient at the interface, using respectively

$$
\overline{T_{i n t}}=\frac{\int_{v} T \phi(1-\phi) \mathrm{d} v}{\int_{v} \phi(1-\phi) \mathrm{d} v}
$$

$$
\overline{\nabla T_{i n t}}=\frac{\int_{v} \nabla T \phi(1-\phi) \mathrm{d} v}{\int_{v} \phi(1-\phi) \mathrm{d} v}
$$

where $\phi(1-\phi)$ allows capturing only the interface region between the two phases. Figure 7 shows the temperature gradient in and around the drop at a given time for our reference simulation. Most heat transfer between the metal drop and the magma ocean occurs at the drop front side, while the back half only accounts for about $15 \%$ of the total. The existence of a hot thermal wake and the external recirculation behind the drop that encapsulates and entrains magma with the sinking drop are the main reasons limiting back thermal exchanges (41; 27).

A close view of the temperature field and of the thermal boundary layer is shown in Figure 8. We define the thermal boundary layer thickness as the distance from the drop interface to a point where the temperature anomaly reaches $1 \%$ of its interfacial value. The boundary layer is very thin at the drop 
front section, where most thermal exchanges are carried out. It significantly increases behind the drop. An example of thickness measurement is shown in Figure 8 (Right) at the thinnest position, i.e. at the front of the drop. In order to give a global estimate around the drop, we also define the average boundary layer thickness as

$$
\delta_{T_{a v}}=\frac{\overline{T_{i n t}}-T_{m}}{\overline{\nabla T_{i n t}}} .
$$

\section{Parametric study and scaling analysis}

We now present our systematic exploration of the parameter space, changing the magma ocean viscosity or the drop initial radius as shown in Table 2 from our reference simulation \#4. We then analyse our results in terms of scaling laws. In particular we determine the influence of the Peclet number on the dimensionless parameters defined in the previous section: the time and length of equilibration, the normalized thermal boundary layer thickness, the Nusselt number, and the dimensionless magma ocean volume affected by thermal exchanges.

\subsection{Time and length of equilibration}

As expected from previous studies of drop dynamics, but largely neglected in geophysical applications (see e.g. discussions in 7, 27), the characteristic time and length of equilibrium depend on the viscosity contrast between the metal drop and the magma ocean. As presented in Figure 9] increasing the viscosity ratio increases the required time for thermal equilibration (Figure 9 Left), mostly because a larger ambient viscosity limits advective heat exchanges. We can observe in this Figure 9 (Left) two different power laws depending on the dynamic regime of the drop. In the first regime (non-deformable drop, $R_{\mu}>1000$ ), the equilibration characteristic time is highly dependent on the viscosity ratio, whereas in the second regime (deformable drop, $R_{\mu}<200$ ), 
the equilibration characteristic time depends slightly on the viscosity ratio and highly on the deformation of the drop. The evolution of the equilibration length is non-monotonic (Figure 9 Right), because the magma ocean viscosity also influences the falling velocity. Globally, with a more viscous ambient fluid, the thermal equilibrium between the two phases occurs less deeply in the magma ocean, as we predicted in our previous work (27).

The drop size at a given viscosity ratio also influences the thermo-chemical equilibration (see 20, for a fixed spherical shape). Increasing the initial radius of the liquid metal drop decreases the surface of exchange over volume initial ratio, and increases the drop falling velocity: we thus expect an increase of both the characteristic time and length, as confirmed in Figure 10 In (27), we predicted that increasing the drop initial radius also leads to surface extension due to drop distortion, hence to faster equilibration compared to a purely spherical drop: this effect is however limited, because drop deformation mostly occurs at the back of the drop, while heat exchanges takes place mostly at the front.

Those two series of results can be rationalized by considering dimensionless properties as a function of the Peclet number. Here, we normalize the equilibrium time by the thermal diffusion time and the equilibrium length by the initial drop radius. Results are shown in Figure 11. The thermal equilibrium time for a high Peclet, spherical drop theoretically scales as $P e^{-0.5}$ (see e.g. 30). Here we find

$$
t_{c}^{*}=2.45 P e^{-0.59 \pm 0.01}
$$

This acceleration of the equilibration compared to the theoretical model may be a signature of the drop deformation at large Peclet. Correspondingly, the length needed to reach the equilibration increases monotonically with Peclet number following

$$
l_{c}^{*}=2.37 P e^{0.41 \pm 0.01},
$$


in agreement with a quasi-constant falling velocity, i.e. $l_{c}=V \times t_{c}$. In conclusion, when the flow advection measured by the Peclet number increases, thermal equilibration occurs faster but deeper in the magma ocean, which is of importance for Earth's building models (31).

\subsection{Heat exchanges}

Simply equilibrating large-scale heat advection along the drop surface by with heat diffusion through the thermal boundary layer perpendicular to it, the theoretical size of the thermal boundary layer normalised by the drop radius classically scales as $P e^{-0.5}$ : this was verified numerically by (20) for a rigid falling sphere. As shown in Figure 12 (Left), we also recover this scaling at the drop front, where most heat exchange occurs: the best fit gives

$$
\delta_{T}^{*}=2.53 P e^{-0.5}
$$

With the deformable drops considered here however, the boundary layer thickness strongly varies around the metal drop: we thus compute a characteristic average value $\delta_{T_{a v}}$ from Eq. 17, normalised by the initial radius. Results are shown in Figure 12 (Right), with the best fit scaling law

$$
\delta_{T_{a v}}^{*}=10.7 P e^{-0.6 \pm 0.01} .
$$

Over the explored range, the average thickness is at least three times larger than the front one. The larger-than-expected 0.6 exponent might again be a signature of drop distortion at large Peclet number.

Then by definition, the Nusselt number averaged over the drop surface should scale as $1 / \delta_{T_{a v}}^{*}$. This is indeed recovered, as shown in Figure 13 with the best fit scaling law

$$
N u=0.08 P e^{0.63 \pm 0.02}
$$


Note that $N u$ varies over time in many cases in this study: we consider here its time-meanaveraged value.

Comparing this result with the one obtained in the previous section, we also recover that the typical dimensionless time for equilibration $t_{c}^{*}$ scales like $1 / N u$, as expected. This proves the self-consistency of our measurements.

\subsection{Volume of heated magma ocean}

In the wake of the drop, the magma ocean is thermally affected by its passage. Because of heat diffusion within the magma ocean, the affected volume widens with time. But on the short times, at a given depth of the drop $z$, it can be estimated by simply balancing the heat that has passed through the drop interface with the heat accumulated in this volume of magma ocean, whose temperature has increased by a given amount larger or equal to $T_{C}$. We then predict

$$
V o^{*}=\frac{\text { Volume of heated magma }}{\text { Initial drop volume }} \sim 3 \frac{N u}{P e} \frac{z}{R} \frac{\Delta T_{0}}{T_{C}} .
$$

with $\Delta T_{0}$ the initial temperature difference between the two phases. Results in Figure 14 show a good agreement with a small relative error for various depths $z=10,20,30 R$ and temperature contrast $T_{C}=1 K$, using the previously determined scaling for $N u$. This indicates the self-coherence of our numerical results from a fluid dynamics point of view through the energy conservation. With this sealing law, we conclude that the mixing in the magma ocean induced by the passage of each drop is very small. Note that here the falling drop encapsulates in its wake a small volume of silicate while the reminder of the ambient fluid remains largely motionlesss or laminar. However, the thermal contamination in the magma ocean induced by the passage of each drop is very small. This volume will always eventually decrease due to thermal diffusion and will always go to zero in a long term due to the absence of viscous dissipation in 
this study. In the context of planetary core formation, the viscous dissipation effect is very important for the release of gravitational potential energy due only to the fall of the drop. Thus, long after the drop has equilibrated with the magma ocean, it still produces a thermal wake as its gravitational potential energy. Thus, in the segregation of the drops, the phenomenon of diffusion of the affected volume of the magma ocean does not always tend towards zero. In addition, the analytical law proposed by the energy balance between the drop and the magma ocean in Eq. 23, is of interest for planet building models by passing from the one drop scenario to a cloud of drops of different sizes resulting from an impactor core with a radius of 10-100 km. Thus, the volume of the total amount of heat exchanged between this cloud and the magma ocean, plays an important role on the global heat budget. Our conclusion should be reevaluated in the presence of global magma motions, coming e.g. from turbulent convection, depending on the typical excited time- and length-scales. But this is beyond the scope of the present paper.

\section{Influence of a temperature dependent viscosity}

The magma ocean viscosity increases from the surface to the base of the magma ocean because of the combined effects of temperature and pressure (21). Those large-scale variations are irrelevant in the context of our local study; nevertheless, during the drop fall over the typical length of $\sim 200 R$ considered here, the temperature of the liquid magma ocean increases because of heat exchanges with the drop, therefore its viscosity decreases at a given pressure (i.e. depth) following the equation of (21) for anhydrous liquid

$$
\mu_{T}=0.00033 e^{(6400 /(T-1000))} \text { Pa.s. }
$$

Here, we investigate the influence of such a temperature dependent viscosity 
on the dynamics, stability and thermal evolution of a drop. For a given initial viscosity ratio, the initial magma ocean temperature is computed using (24), and we then consider an initial temperature difference with the hot metal drop of $\Delta T=100 \mathrm{~K}$ or $\Delta T=1000 \mathrm{~K}$. We focus on 3 reference cases, only changing the viscosity ratio (see Table 2). Those cases are first presented in the absence of temperature dependent viscosity:

- The non-deformable drop case (simulation \#3 in Table 2, $R e=0.76$ and $\mathrm{Pe}=254$ ) is presented in Figure 15. Drop's shape remains mostly constant. The temperature is close to uniform inside the drop because of internal recirculation, and progressively decreases in the wake. Most heat transfer takes place in the front.

- The weakly deformable drop case (simulation \#5 in Table 2 , Re=21.8 and $\mathrm{Pe}=728.36$ ) is presented in Figure 16. The drop quickly deforms towards a spherical cap, associated with a strong release of heat in its wake. The system then reaches a quasi-steady state, with the most surprising feature being a more rapid temperature decrease in the drop than in the wake, leading to a positive heat transfer from the silicate to the iron in the drop's back. Nevertheless, most heat transfer still takes place at the front.

- Finally, the strongly deformable drop case (simulation \#6 in Table 2 $\mathrm{Re}=47.6$ and $\mathrm{Pe}=793.73)$ is presented in Figure 17. Here, the drop shape keeps oscillating over the whole depth because of competing surface tension and inertial forces. This induces oscillatory temperature changes in the wake, associated to periodic thermal plumes emitted from the drop sides, and strong inhomogeneous fluxes within the magma ocean. Nevertheless heat transfers between iron and silicate are still largely focused at the drop's front, explaining while this case is not associated with any specific signature in the previous section. 

$475 \quad 17$.

We now re-run those three cases using the previously defined temperaturedependent viscosity.

\subsection{Drop dynamics}

Figure 18 shows the normalized average velocity of each drop as a function of the normalized time for constant magma ocean viscosity in black lines and for temperature dependent viscosity with $\Delta T=100 \mathrm{~K}(\Delta T=1000 \mathrm{~K})$ in red (blue) dashed lines. The normalized average velocity for the non-deformable drop (Figure 18 Left) with $\Delta T=100 \mathrm{~K}$, increases moderately by about $4.8 \%$ compared to the constant viscosity case, keeping the spherical shape without change in the morphology. For $\Delta T=1000 \mathrm{~K}$, the drop deforms from the back side and its velocity strongly increases by about $26 \%$; concomitantly, the average viscosity around the drop decreases by $93 \%$, explaining this significant dynamical change. On the contrary, for the weakly deformable drop (Figure 18 Middle) and the strongly deformable drop (Figure 18 Right), no significant dynamical change is observed, despite a large viscosity decrease in the associated thermal boundary layer (by $76 \%$ and $92 \%$, respectively, for $\Delta T=1000 \mathrm{~K}$; see also Figure 19. This indicates that the drop dynamics is already mostly inviscid in the absence of temperature-dependent viscosity, as can be guessed from the corresponding values of the Reynolds number (see caption of Figures 16 and

\subsection{Drop fragmentation}

In the most extreme previous case (the strongly deformable drop with $\Delta T=$ 1000 K), Figure 19 indicates that the hot silicate layer surrounding the drop has a viscosity value of the order 0.05 Pa.s: assuming such a viscosity uniformly in the ambient magma ocean, our previous study (27) predicts a rapid fragmentation, which is not observed here. Actually the viscosity constrast at the interface 
does not influence the drop stability, because the thermal boundary layer where the low viscosity is localised is very thin in front of the drop, compared to any relevant dynamical length scale; besides, the wake has no influence on the drop. Temperature dependent viscosity only affects the drop's dynamics and fragmentation when flows and temperature change on comparable scales, in upfront or sides locations.

To further prove this, we performed a simulation with a drop radius of 25 $\mathrm{mm}$ and a viscosity ratio of 200 for constant magma ocean viscosity first, then for $\Delta T=1000 \mathrm{~K}$ dependent magma ocean viscosity (simulation \#10 in Table 2). Figure 20 (Left) shows the Jellyfish fragmentation mode when constant magma ocean viscosity is considered (more details can be found in 27) . The normalized time $\left(t_{b k}^{*}=\frac{t_{b k} V}{2 R} \sqrt{\frac{\rho_{d}}{\rho_{m}}}\right)$ and distance $\left(d_{b k}^{*}=\frac{d_{b k}}{R}\right)$ of breakup equal to 3.8 and 10.7 respectively. For temperature-dependent viscosity (Figure 20 middle), another Jellyfish fragmentation mode is observed, with time and distance of breakup equal to 2.3 and 5.6 respectively. Actually, here, the extended jellyfish membranes form filaments, where thermal effects are very important. These membranes sink into a less viscous medium whose thickness is comparable to the membranes thickness (see Figure 20 Right). So, the inertia forces in this zone are stronger than the surface tension, and the fragmentation occurs. Thus, the filaments move freely in this zone with low viscous constraint and therefore separate from the drop volume leading to the fragmentation.

Note finally that the local dimensionless numbers, taken at the mean temperature (and associated viscosity) of the thermal boundary layer, become $R e=1000, W e=19$ and $R_{\mu}=10$. From Figures 4, 12 and 13 of our previous study (27), we find consistent results for the fragmentation mode of the drop, as well as for its time and distance of breakup, accounting for these dimensionless numbers (To compare with our previous study, $R e$ and $W e$ should 
be multiplied in this study by a factor of 2 because in (27), Re and $W e$ are calculated by the drop diameter).

Hence, temperature dependent magma ocean viscosity influences the drop fragmentation in the filament forming regimes only. In such regimes, the results in (27) as a function of $R e, W e$ and $R_{\mu}$ already allow predicting fragmentation mode, breaking time and distance by considering local values of the dimensionless numbers.

\subsection{Thermal evolution}

In Figure 21, we compare the normalized mean iron temperature for each case as a function of normalized time. For a non-deformable drop (Figure 21 Left), the average temperature for $\Delta T=100 \mathrm{~K}$ (red dashed lines) and $\Delta T=1000 \mathrm{~K}$ (blue dashed lines) temperature-dependent viscosity decreases by about $6.1 \%$ and $20.8 \%$ with respect to the constant viscosity case (black line). As a consequence, it significantly changes the characteristic time and length of equilibration (see Table 3). For a weakly deformable drop (Figure 21 Middle), the average temperature evolution with $\Delta T=1000 \mathrm{~K}$ changes only by about $13.6 \%$; no difference is measured for a strongly deformable drop (Figure 21 Right). As for the fall velocity, temperature dependent viscosity influences significantly the thermal evolution of non-deformable drops only (i.e. the drops in the Stokes regime). Note finally that in the intermediate regime, the characteristics time and length of equilibration decrease slightly with a temperature dependent viscosity compared to constant magma ocean viscosity case, whereas in the Stokes regime, the equilibrium characteristic length increases with a temperature dependent viscosity, in contrast to the characteristic time of equilibration (see Table 3).

As for the volume of heated magma ocean shown in Figure 22, no significant change is observed in the heated volume for all cases with $\Delta T=100 \mathrm{~K}$ (red 
dashed lines). The volume of heated magma ocean increases by about 1.5 to 2 times compared to the constant viscosity case (black line) for $\Delta T=1000 \mathrm{~K}$ (blue dashed lines) due to the initial larger temperature difference; but the curves remain parallel, hence exhibiting no specific dynamical signature.

Table 3: The change in characteristics time and length for cases of temperature-dependent viscosity.

\begin{tabular}{lccc}
\hline Simulation & $\Delta T$ & $t_{c}^{*}$ & $l_{c}^{*}$ \\
\hline \#3 (constant magma ocean viscosity $)$ & 100 & 0.1 & 24.25 \\
\hline \#3a $\left(T\right.$ dependent $\left.\mu_{m}\right)$ & 100 & 0.0975 & 25.6 \\
\hline \#3b $\left(T\right.$ dependent $\left.\mu_{m}\right)$ & 1000 & 0.082 & 27 \\
\hline \#5 (constant magma ocean viscosity) & 100 & 0.05 & 35.14 \\
\hline \#5a $\left(T\right.$ dependent $\left.\mu_{m}\right)$ & 100 & 0.0496 & 33.33 \\
\hline \#5b $\left(T\right.$ dependent $\left.\mu_{m}\right)$ & 1000 & 0.045 & 31.25 \\
\hline \#6 (constant magma ocean viscosity) & 100 & 0.045 & 32 \\
\hline \#6a $\left(T\right.$ dependent $\left.\mu_{m}\right)$ & 100 & 0.044 & 29.4 \\
\hline \#6b $\left(T\right.$ dependent $\left.\mu_{m}\right)$ & 1000 & 0.042 & 27.7 \\
\hline
\end{tabular}

Finally, we calculate the heat transfer between the two phases "Nusselt number" of all drops in this section for a temperature dependent viscosity. Then, we compare these results with the correlations proposed in the experimental and numerical studies of the penetration of a hot diapir through a highly temperaturedependent viscous medium $(42,43)$. These correlations of the Nusselt number are done for non-deformable spherical drops of low Reynolds number and are given as follows:

$$
N u=1+C_{1} P e^{1 / 3}
$$

where $C_{1}=0.45 \pm 0.09$.

$$
N u=0.795+0.459\left[\operatorname{Pe}\left(\left(\frac{\Delta T}{\ln 10}\right)^{3} \frac{\overline{\mu_{\text {int }}}}{\mu_{m}}\right)^{-1 / 5}\right]^{1 / 2}
$$

54 The Eq. 25 has been proposed experimentally by (42) and the Eq. 26 has been proposed numerically by (43). The comparison of the Nusselt numbers between 
our numerical results and the Eqs. 22, 25 and 26 is shown in Table 4.

Table 4: The comparison of the Nusselt numbers for cases of temperature-dependent viscosity between our numerical results and the Eqs. 22, 25, and 26

\begin{tabular}{lcrrc}
\hline Simulation & Nu: this study & Nu: Eq. 22 & Nu: Eq. 25 & Nu: Eq. 26 \\
\hline \#3a & 2.65 & 2.72 & $3.91 \pm 0.58$ & 3.36 \\
\hline \#3b & 2.95 & 3.07 & $4.10 \pm 0.62$ & 2.82 \\
\hline \#5a & 5.15 & 5.17 & $5.08 \pm 0.82$ & 4.93 \\
\hline \#5b & 5.21 & 5.33 & $5.15 \pm 0.83$ & 3.65 \\
\hline \#6a & 5.28 & 5.36 & $5.16 \pm 0.83$ & 5.06 \\
\hline \#6b & 5.35 & 5.36 & $5.16 \pm 0.83$ & 4.58 \\
\hline
\end{tabular}

550

We observe that our numerical results are consistent with Eq. 26 for the non-deformable drops and with Eq. 25 for the deformable drops. This proves the validity of our calculations in this section. We further note that our Nusselt number scaling law in section 4.2 (Eq. 22) is valid for this section of a temperature-dependent viscosity.

\section{Conclusions and future work}

We have carried out series of numerical simulations to characterize the thermal exchanges between a falling drop and a viscous ambient fluid, exploring a new parameter range relevant for the geophysical application of a hot liquid iron drop falling in a magma ocean. We have shown that because of drop distortions, thermal equilibration properties slightly change from the theoretical predictions based on diffusive heat exchanges through a laminar thermal boundary layer. We have also tested that accounting for a temperature-dependent viscosity in the magma ocean barely influences the obtained results, except for limited cases like non-deformable, low Reynolds number drops or Jellyfish fragmentation mode. Our most relevant results for geophysical application are the scaling laws for the normalized length of equilibration and for the Nusselt number, which both increase monotonically with the Peclet number as $P e^{0.41}$ and $P e^{0.63}$, respectively. 
Future work should now consider (i) the presence of convection in the ambi570 ent magma ocean, which could affect our conclusions providing the associated 571 velocity at the drop scale is at least of similar order as the falling drop velocity, 572 hence requiring extremely turbulent regimes; and (ii) chemical exchanges be573 tween iron and silicates, which will determine the initial chemical state of the 574 considered planet (see e.g. 44). Chemical and thermal constitutive equations 575 being similar, similar equilibration scaling laws and dynamics are nevertheless 576 expected. 
Acknowledgements

The authors acknowledge funding by the ERC under the European Union's Horizon 2020 research and innovation program through Grant No. 681835FLUDYCO-ERC-2015-CoG. This research also received funding from the French PNP program (INSU-CNRS), the French Government Laboratory of Excellence initiative No. ANR-10-LABX-0006, and the Région Auvergne. This paper is Laboratory of Excellence ClerVolc contribution no. XX. We are grateful to the Mésocentre Clermont Auvergne University for providing help as well as computing and storage resources. 


\section{References}

[1] T. Kleine, C. Münker, K. Mezger, and H. Palme. Rapid accretion and early core formation on asteroids and the terrestrial planets from Hf-W chronometry. Nature, 418:952-955, 2002.

[2] M. Touboul, T. Kleine, B. Bourdon, H. Palme, and R. Wieler. Late formation and prolonged differentiation of the Moon inferred from $\mathrm{W}$ isotopes in lunar metals. Nature, 450:1206-1209, 2007.

[3] D. J. Stevenson. fluid dynamics of core formation. Origin of the Earth, pages 231-249, 1990.

[4] R. Deguen, P. Olson, and P. Cardin. Experiments on turbulent metalsilicate mixing in a magma ocean. Earth Planet. Sci. Lett., 310:303-313, 2011.

[5] R. Deguen, M. Landeau, and P. Olson. Turbulent metal - silicate mixing, fragmentation, and equilibration in magma oceans. Earth Planet. Sci. Lett., 391:274-287, 2014.

[6] M. Landeau, R. Deguen, and P. Olson. Experiments on the fragmentation of a buoyant liquid volume in another liquid. Fluid Mech., 749:478518, 2014 .

[7] J. B. Wacheul, M. LeBars, J. Monteux, and J. M. Aurnou. Laboratory experiments on the breakup of liquid metal diapirs. Earth Planet. Sci. Lett., 403:236245, 2014.

[8] J. B. Wacheul and M. Le Bars. Experiments on fragmentation and thermochemical exchanges during planetary core formation. Phys. Earth Planet. Int., 276:134 144, 2018. 
[9] W. K. Hartmann and D. R. Davis. Satellite-sized planetesimals and lunar origin. Icarus, 24:504514, 1975.

[10] W. B. Tonks and H.J. Melosh. Core formation by giant impacts. ICARUS, 100:326-346, 1992.

[11] J. Monteux, N. Coltice, F. Dubuffet, and Y. Ricard. Thermo-mechanical adjustment after impacts during planetary growth. Geophys. Res. Lett., 34:24201-24205, 2007.

[12] M. J. Walter and R. G. Tronnes. Early earth differentiation. Earth Planet. Sci. Lett., 225:253 269, 2004.

[13] J. Monteux, Y. Ricard, N. Coltice, F. Dubuffet, and M. Ulvrova. A model of metal-silicate separation on growing planets. Earth and Planetary Science Letters, 287:353-362, October 2009.

[14] J. D. Kendall and H. Melosh. Differentiated planetesimal impacts in a terrestrial magma ocean: fate of the iron core. Earth Planet. Sci. Lett., 448:2433, 2016.

[15] H. Genda, R. Brasser, and S.J. Mojzsis. The terrestrial late veneer from core disruption of a lunar-sized impactor. Earth and Planetary Science Letters, 480:2532, 2017.

[16] D. C. Rubie, H. J. Melosh, J. E. Reid, C. Liebske, and K. Righter. Mechanisms of metal-silicate equilibration in the terrestrial magma ocean. Earth and Planet. Sci. Lett., 205:239-255, January 2003.

[17] H. Ichikawa, S. Labrosse, and K. Kurita. Direct numerical simulation of an iron rain in the magma ocean. GEOPHYSICAL RESEARCH, 115:B01404, 2010. 
[18] H. Samuel. A re-evaluation of metal diapir breakup and equilibration in terrestrial magma oceans. Earth Planet. Sci. Lett., 313-314:105-114, 2012.

[19] T. W. Dahl and J. Stevenson. Turbulent mixing of metal and silicate during planet accretion and interpretation of the hf-w chronometer. Earth Planet. Sci. Lett., 295:177 - 186, 2010.

[20] M. Ulvrová, N. Coltice, Y. Ricard, S. Labrosse, and F. Dubuffet Velìmskỳand M. Šràmek. Compositional and thermal equilibration of particles, drops, and diapirs in geophysical flows. Geochem. Geophys. Geosyst, $12,2011$.

[21] B. B. Karki and L. P. Stixrude. Viscosity of mgsio3 liquid at earthś mantle conditions : Implications for an early magma ocean. Science, 328:740-742, 2010.

[22] T. Bonometti and J. Magnaudet. Transition from spherical cap to toroidal bubbles. Phys. Fluids, 18:052102, 2006.

[23] M. Ohta, Y. Akama, Y. Yoshida, and M. Sussman. Three-dimensional simulations of vortex ring formation from falling drops in an immiscible viscous liquid. Chem. Engng Japan, 42:648655, 2009.

[24] M. Ohta, S. Yamaguchi, Y. Yoshida, and M. Sussman. The sensitivity of drop motion due to the density and viscosity ratio. Phys. Fluids, 22:072102, 2010.

[25] M. Ohta, Y. Akama, Y. Yoshida, and M. Sussman. Inuence of the viscosity ratio on drop dynamics and breakup for a drop rising in an immiscible low-viscosity liquid. Fluid Mech., 752:383409, 2014.

[26] M. Ohta and M. Sussman. The buoyancy-driven motion of a single skirted 
bubble or drop rising through a viscous liquid. Phys. Fluids, 24:112101, 2012.

[27] B. Qaddah, J. Monteux, V. clesi, A.Bouhifd, and M. Le Bars. Dynamics and stability of an iron drop falling in a magma ocean. Phys. Earth Planet. Int., 289:75 89, 2019.

[28] X-Y. Luo, M-J. Ni, A. Ying, and M. Abdou. Application of the level set method for multi-phase flow computation in fusion engineering. Fusion Engineering and Design, 81:15211526, 2006.

[29] J. Hu, R. Jia, K.T. Wan, and X. Xiong. Simulation of droplet impingement on a solid surface by the level set method. COMSOL Conference in Boston, 2014 .

[30] V. Lherm and R. Deguen. Small-scale metal/silicate equilibration during core formation: The inuence of stretching enhanced diusion on mixing. Journal of Geophysical Research : Solid Earth, 123:10.49610.516, 2018.

[31] D.C. Rubie, F. Nimmo, and H.J Melosh. Formation of the earths core, treatise on geophysics evolution of the earth, second ed. Elsevier, 9:43-79, 2015.

[32] A. M. Hofmeister. Mantle values of thermal conductivity and the geotherm from phonon lifetimes. SCIENCE, 283, 1999.

[33] C. W. Thomas and P.D. Asimow. Direct shock compression experiments on premolten forsteriteand progress toward a consistent highpressure equationof state for cao-mgo-al2o3-sio2-feo liquids. JOURNAL OF GEOPHYSICAL RESEARCH: SOLID EARTH, 118:5738-5752, 2013.

[34] N. de Koker, G. Steinle-Neumann, and V. Vlček. Electrical resistivity and 
thermal conductivity of liquid fe alloys at high $\mathrm{p}$ and $\mathrm{t}$, and heat flux in earths core. PNAS, 109:4070-4073, 2012.

[35] H. Ichikawa, T. Tsuchiya, and Y. Tange. The p-v-t equation of state and thermodynamic properties of liquid iron. Journal of Geophysical Research: Solid Earth, 119:240-252, 2014.

[36] M. Pilch and C. A. Erdman. Use of breakup time data and velocity history data to predict the maximum size of stable fragments for accelerationinduced breakup of a liquid drop. Multiphase Flow, 13:741757, 1987.

[37] E. Villermaux and B. Bossa. Single - drop fragmentation determines size distribution of rain drops. Nat. Phys., 5:697702, 2009.

[38] S.S Jain, N. Tyagi, R. S. Prakash, R.V Ravikrishna, and G. Tomar. Secondary breakup of drops at moderate weber numbers: Eect of density ratio and reynolds numbe. Int. J. Multiphase Flow, 117:2541, 2019.

[39] J. Crank. The mathematics of diffusion, 2nd edition. Clarendon Press, Oxford, 1975.

[40] R.C. Mittal and R.K. Jain. Redefined cubic b-splines collocation method for solving convectiondiffusion equations. Applied Mathematical Modelling, $36: 5555-5573,2012$.

[41] J.R. Fleck, C.L. Rains, D.S. Weeraratne, C.T. Nguyen, D.M. Brand, S.M. Klein, J.M. McGehee, J.M. Rincon, C. Martinez, and P.L. Olson. Iron diapirs entrain silicates to the core and initiate thermochemical plumes. Nature Communications, 2018.

[42] N. M. Ribe. Diapirism in the earth's mantle: Experiments on the motion of a hot sphere in a fluid with temperature-dependent viscosity. Journal of Volcanology and Geothermal Research, 16(3):221 - 245, 1983. 
[43] S. F. Daly and A. Raefsky. On the penetration of a hot diapir through a strongly temperature-dependent viscosity medium. Geophysical Journal International, 83:657681, 1985.

[44] M. Bouhifd and A. Jephcoat. The effect of pressure on partitioning of ni and co between silicate and iron-rich metal liquids: a diamond-anvil cell study. Earth Planet. Sci. Lett., 209:245255, 2003. 

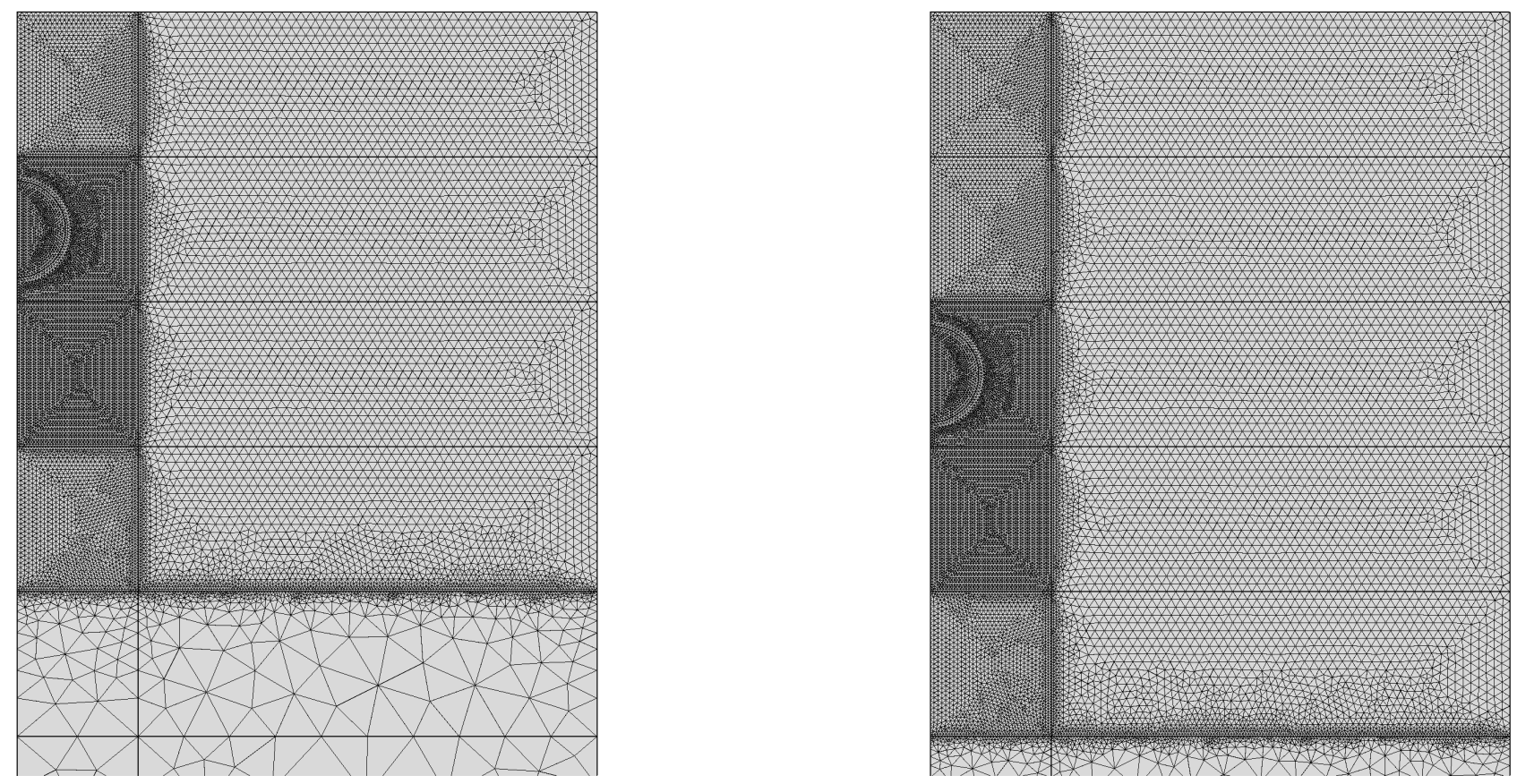

Figure 1: A zoom illustrating our adaptive mesh (left) and the method for mesh evolution over time when the drop reaches the bottom of the finest mesh region (right). 

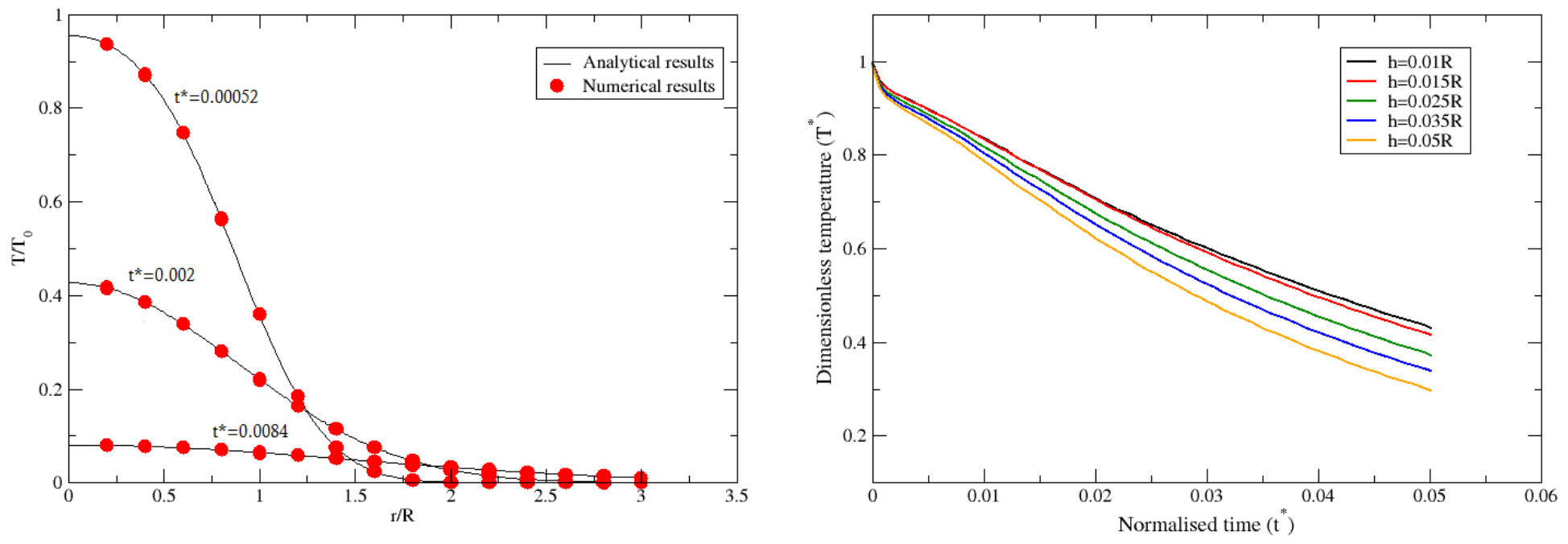

Figure 2: Left: comparison of our numerical results with the analytical results of (39) for the radial profile of the normalised temperature anomaly for a non-deformable and immobile drop. Right: comparison of the time evolution of the normalised mean temperature anomaly determined with different mesh sizes for a deformable and mobile drop (simulation \#4 in Table 2p. 

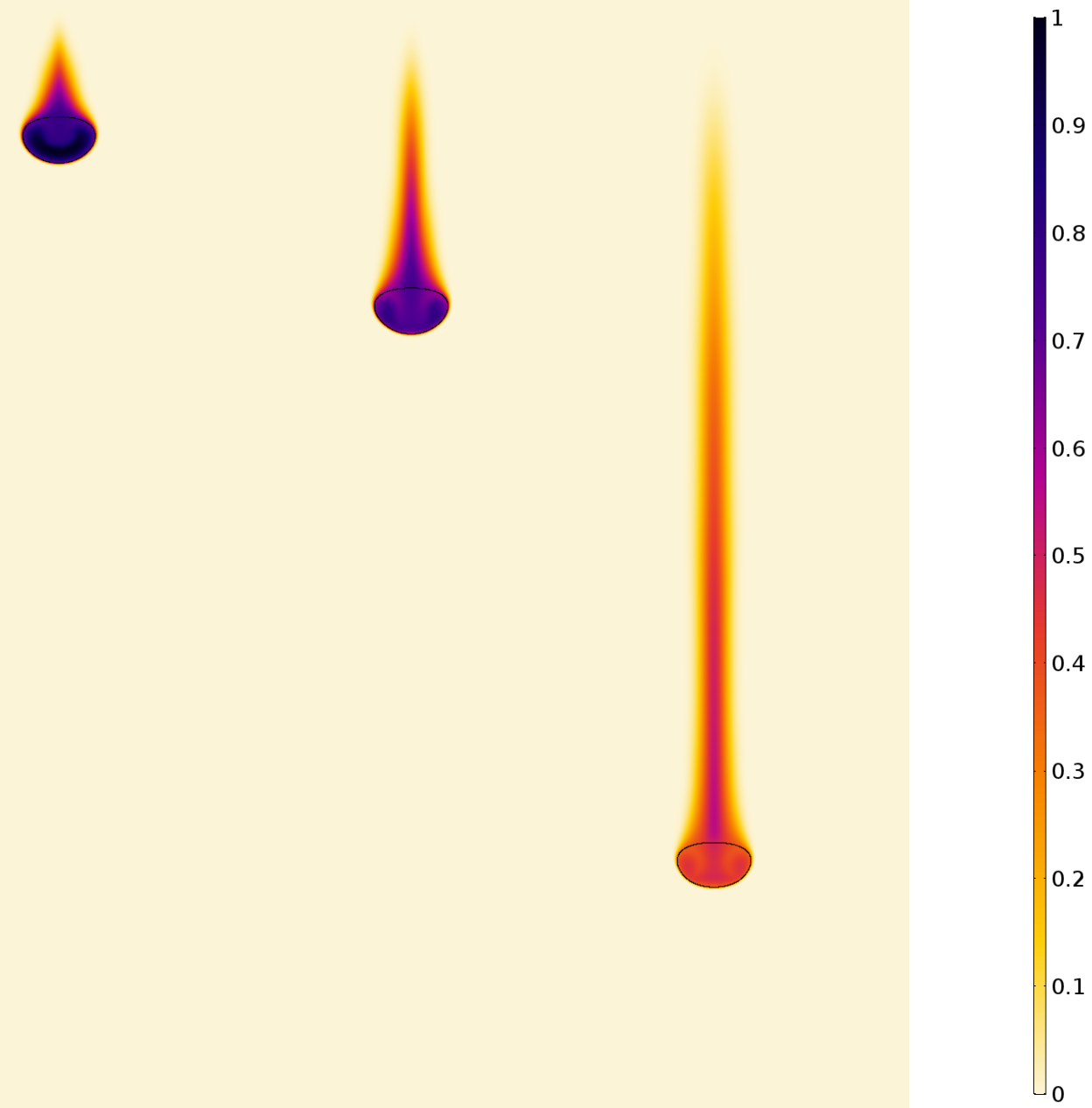

Figure 3: Dynamical and thermal evolutions of the metal drop as a function of time for simulation \#4 in Table 2 The color presents the temperature anomaly normalised by its initial value $T^{*}$. The black solid line separates the metallic material from the magma ocean. From left to right, the time normalised by the thermal diffusion time is $t^{*}=0,0.01,0.02$ and 0.05 . 


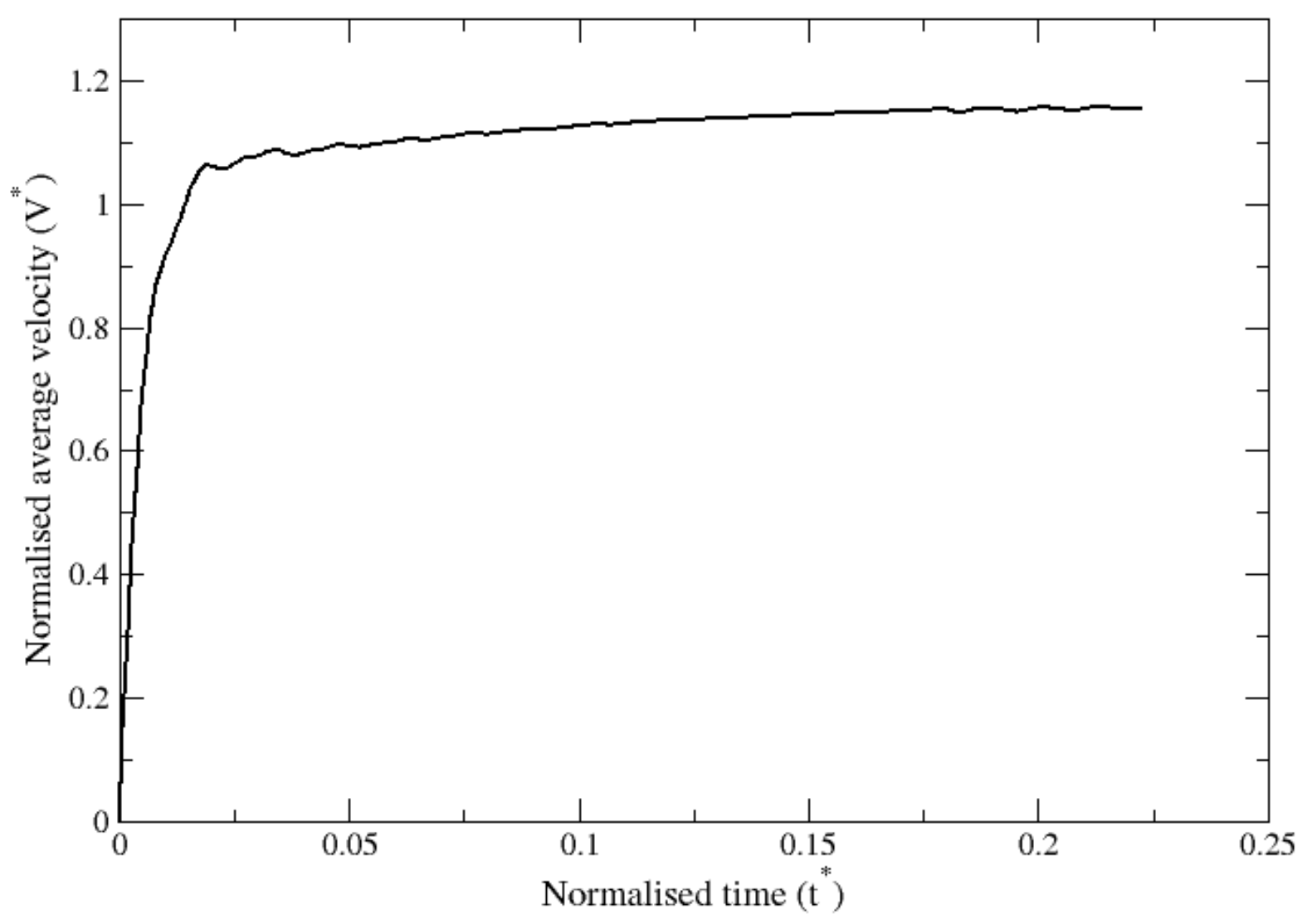

Figure 4: Normalized average velocity of the metal drop as a function of normalized time for simulation \#4 in Table 2 

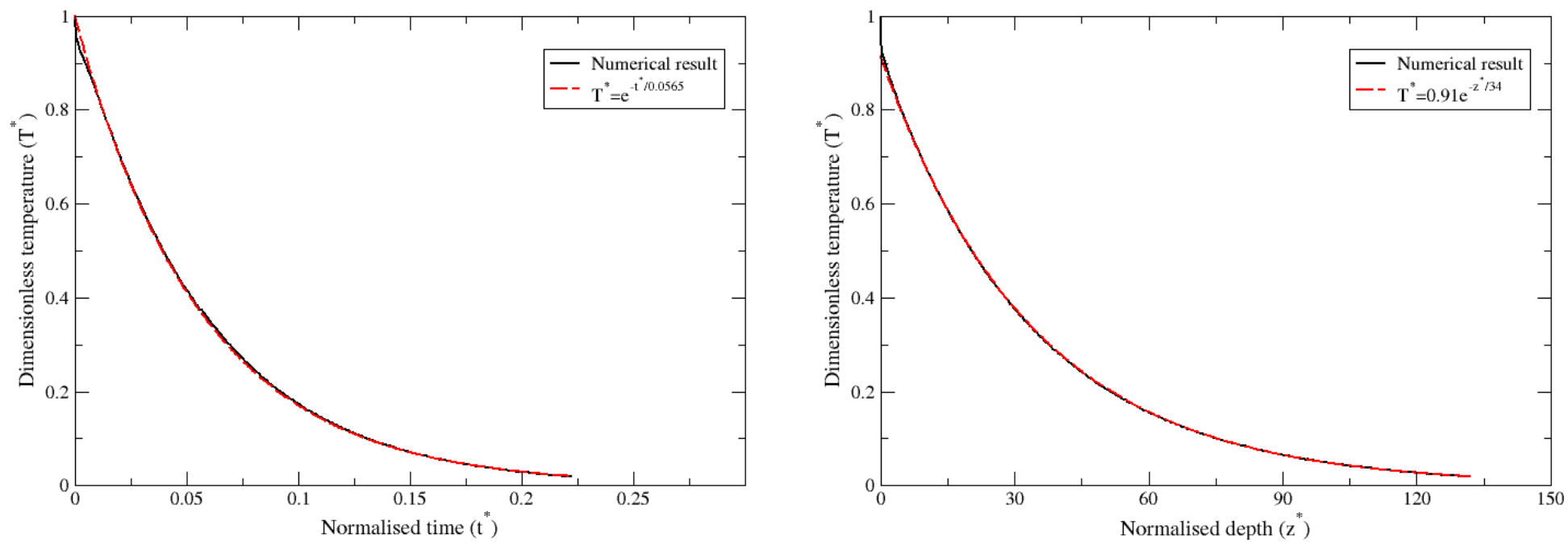

Figure 5: Normalized average temperature anomaly of the metal drop as a function of normalized time (left) and depth (right) for simulation \#4 in Table 2 The black lines represent our numerical results and the red dashed lines represent the exponential fits from Eq. 13 The correlation coefficient for the two fits is 0.9999 . 

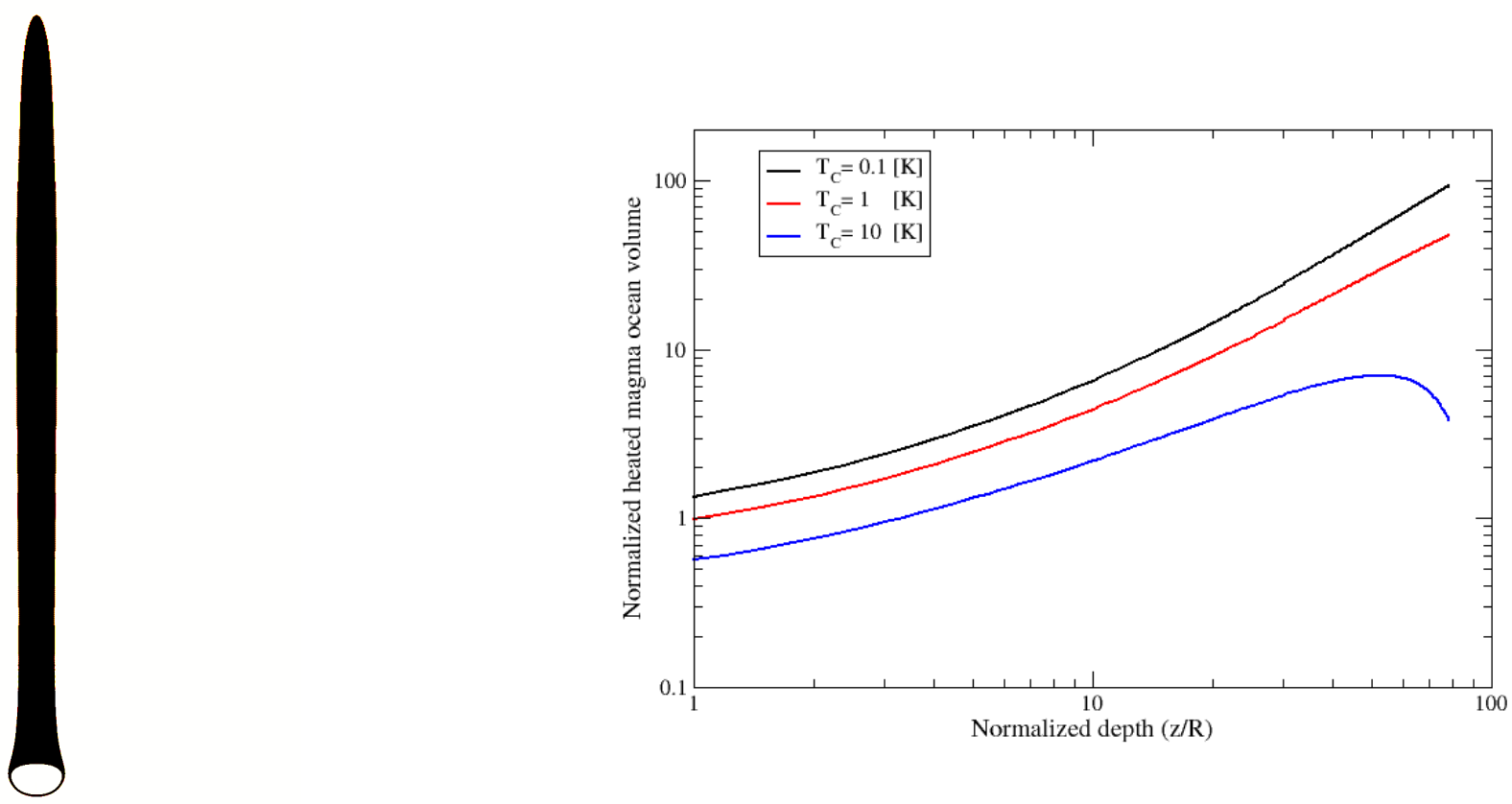

Figure 6: Left: volume of heated magma ocean $\left(T_{C}=1 \mathrm{~K}\right)$ once the drop reaches a time $t^{*}=0.067$ and a depth $z=37 R$ in our simulation \#4 in Table 2 Right: normalized heated magma volume as a function of normalized depth for different values of $T_{C}$. 


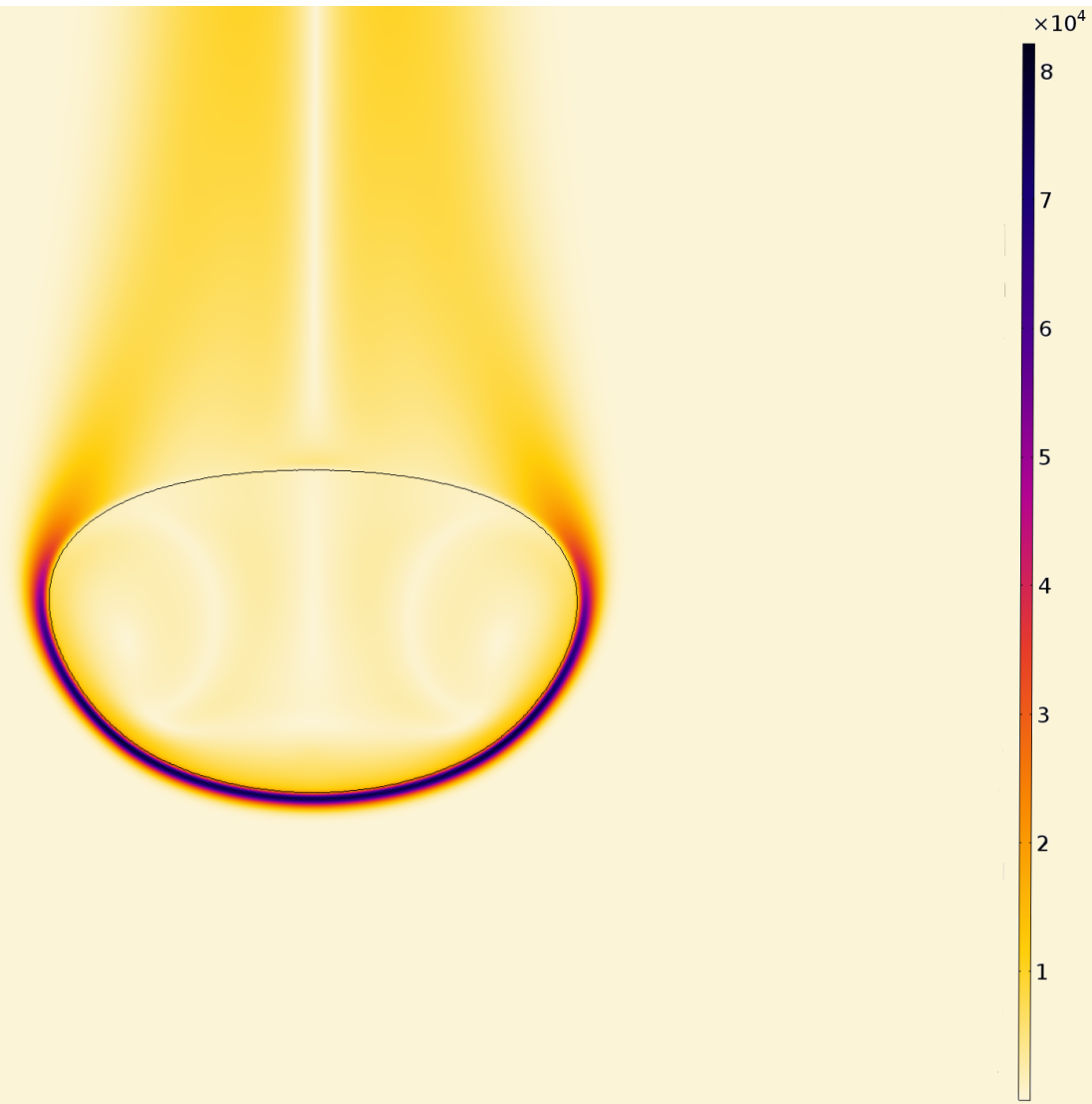

Figure 7: Temperature gradient $(K / m)$ in and around the drop for our reference simulation \#4 in Table 2 at time $t^{*}=0.055$. 

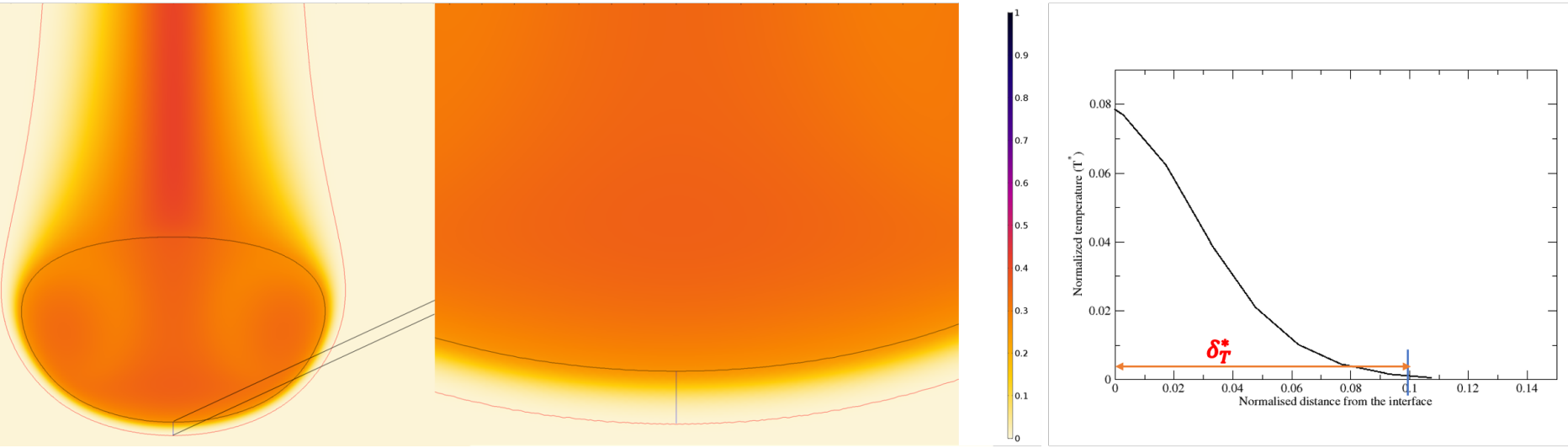

Figure 8: Left: temperature anomaly (colorscale) and contour of the thermal boundary layer (red line) around the drop (black line) for our reference simulation \#4 in Table 2 at time $t^{*}=0.067$. Middle: zoom at the drop front. Right: temperature profile of the thermal boundary layer in front of the drop and determination of the local boundary layer thickness. 

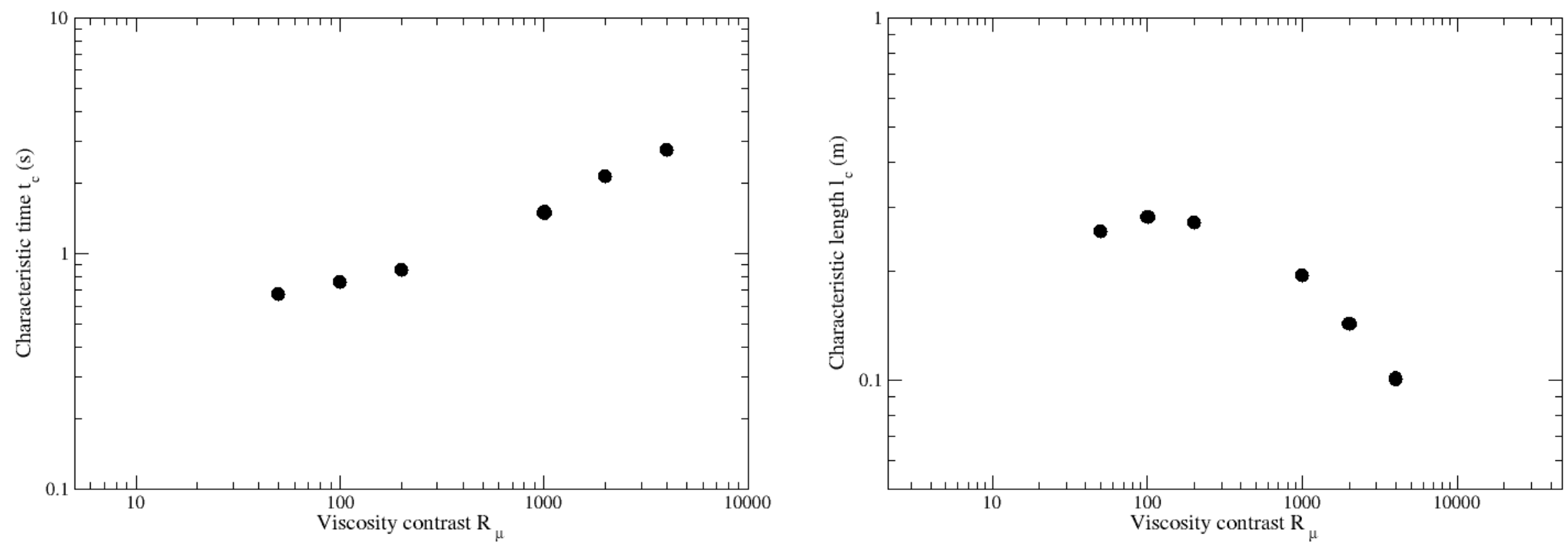

Figure 9: Characteristic time (Left) and length (Right) of equilibration as a function of the viscosity contrast (see Eq. 113). 

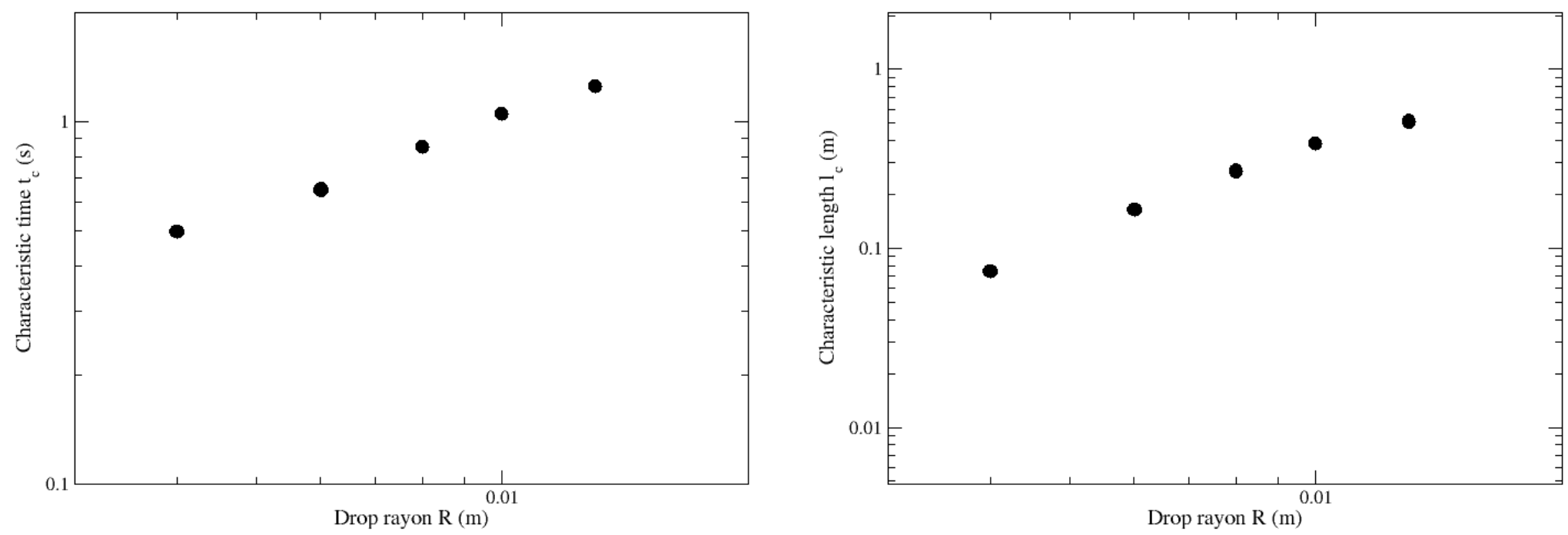

Figure 10: Characteristic time (Left) and length (Right) of equilibration as a function of the drop initial radius (see Eq. 13). 

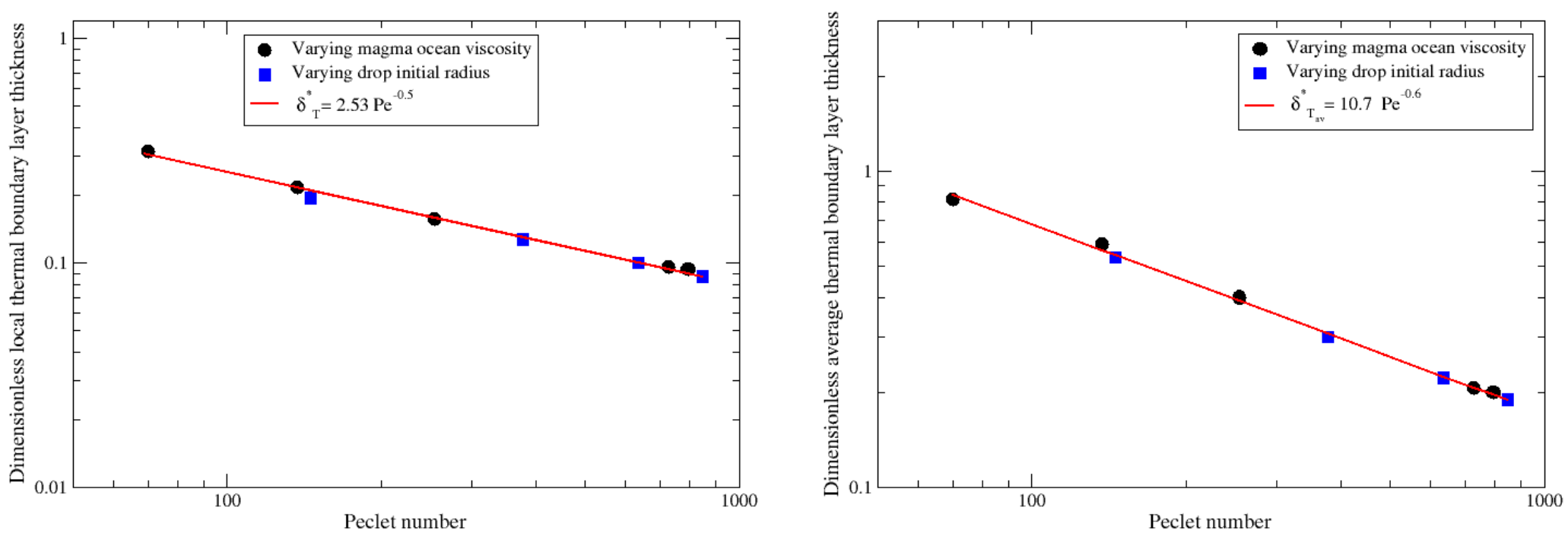

Figure 12: Dimensionless thickness of the thermal boundary layer at the drop front as a function of Peclet number (Left). Dimensionless average thickness of the thermal boundary layer around the drop as a function of Peclet number (Right). 


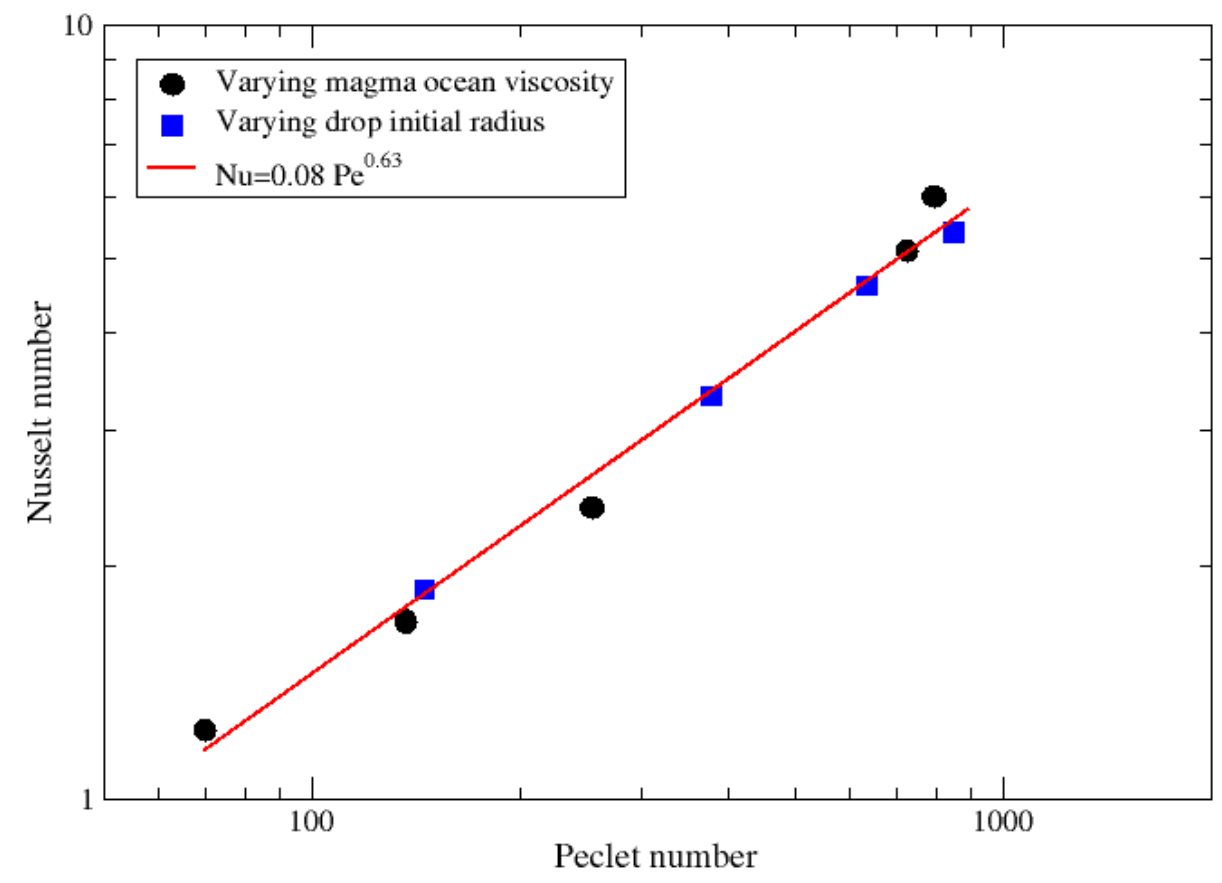

Figure 13: Nusselt number as a function of Peclet number 


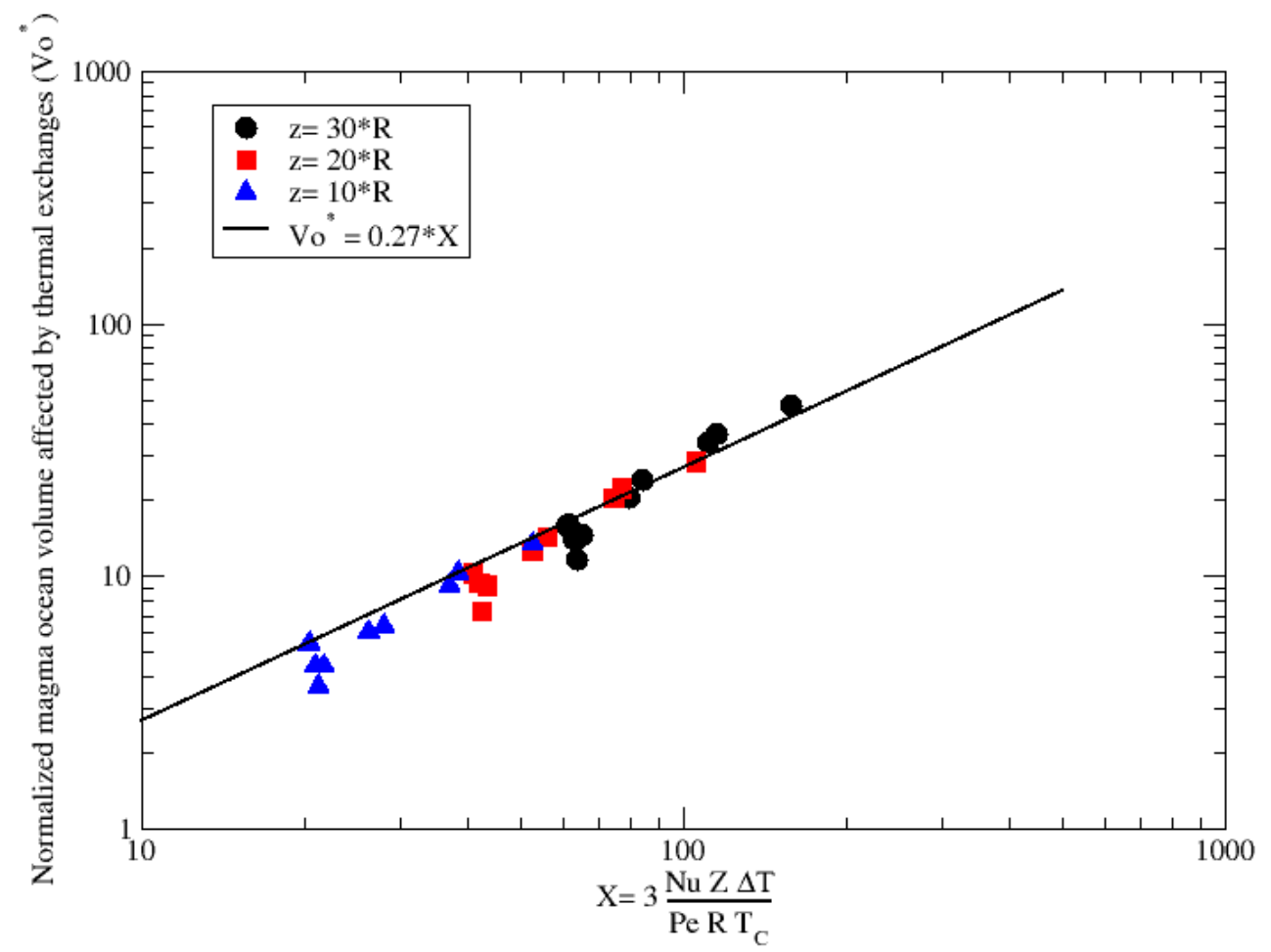

Figure 14: Dimensionless heated magma ocean volume as a function of $X$, and comparison with our scaling law 23 . Our numerical results are represented with different symbols for different given depths. 

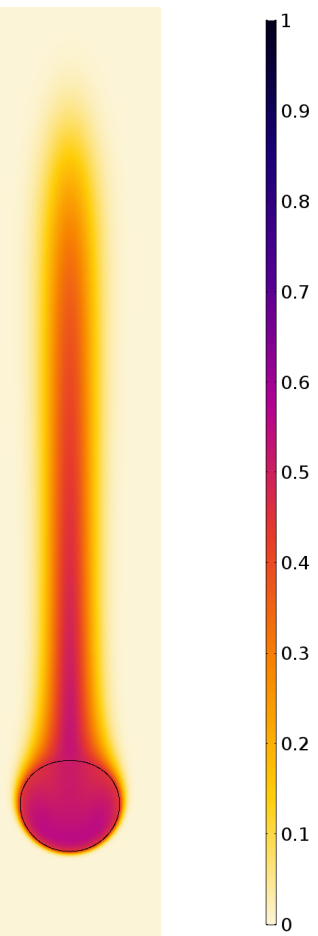

Figure 15: Thermal evolution of the non-deformable metal drop as a function of normalized time (Left). From left to right, $t^{*}=0,0.0167,0.033,0.05$ and 0.067 . The right figure shows the temperature gradient $(\mathrm{K} / \mathrm{m})$ in and around the spherical drop case. In this model, $R e=0.76$, $P e=254$ and $R_{\mu}=1000$ (simulation \#3 in Table 2) 

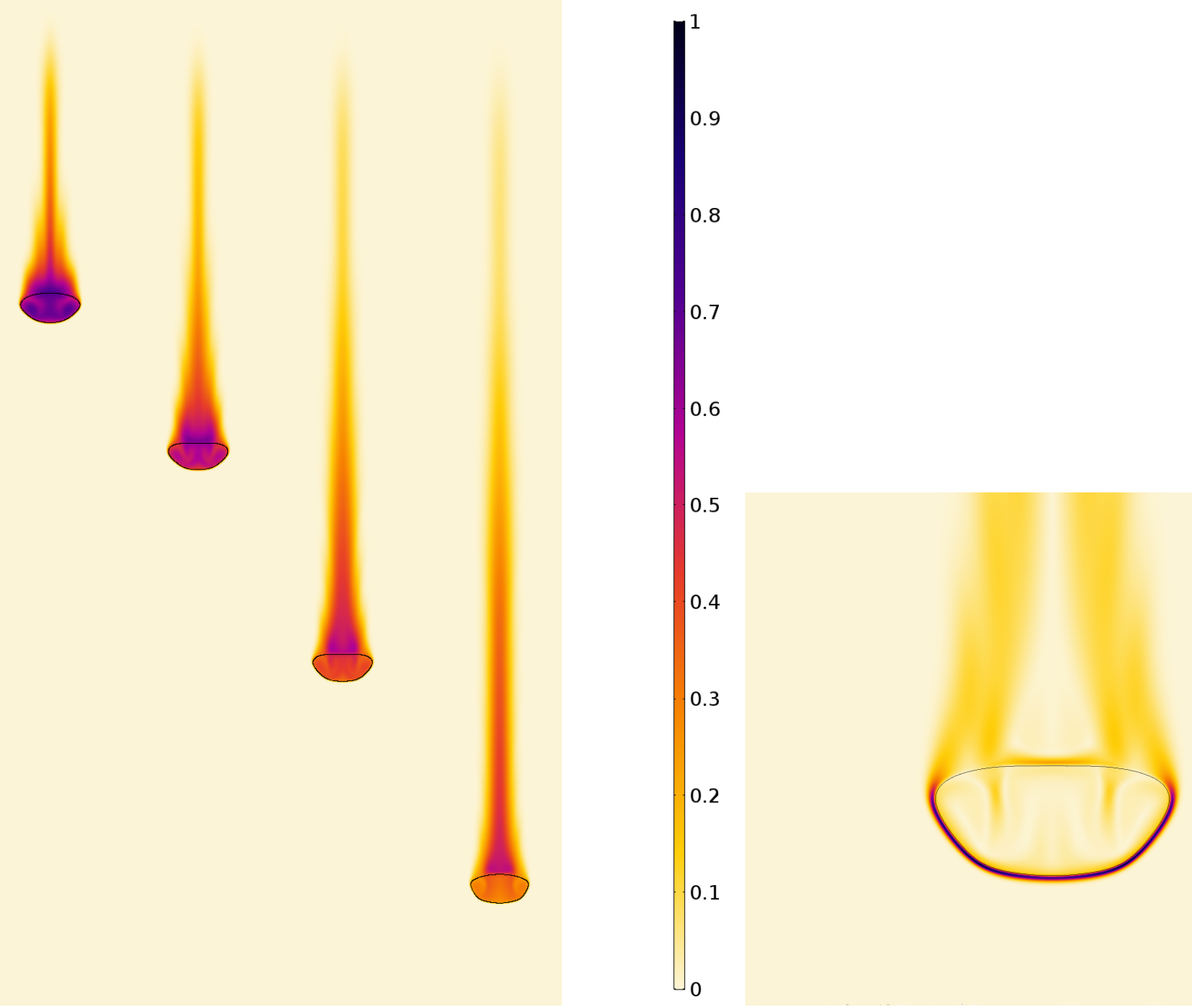

Figure 16: Thermal evolution of the weakly deformable metal drop as a function of normalized time (Left). From left to right, $t^{*}=0.015,0.024,0.033,0.047$ and 0.06 . The right figure shows the temperature gradient $(\mathrm{K} / \mathrm{m})$ in and around the deformable drop. In this model, $R e=21.8$, $P e=728.36$ and $R_{\mu}=100$ (simulation \#5 in Table 2) 

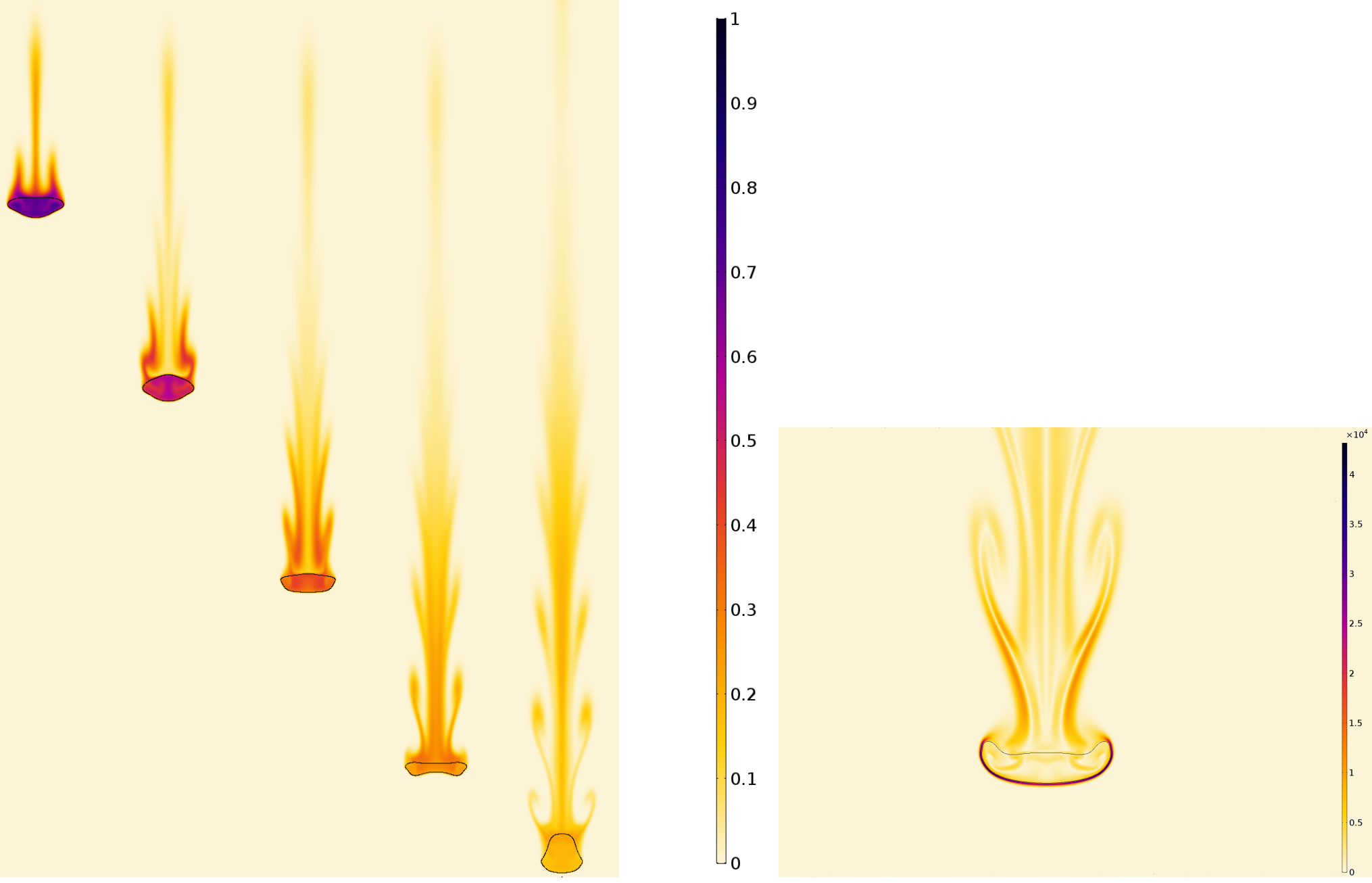

Figure 17: Thermal evolution of the strongly deformable metal drop as a function of normalized time (Left). From left to right, $t^{*}=0.02,0.033,0.047,0.06$ and 0.077 . The right figure shows the temperature gradient $(\mathrm{K} / \mathrm{m})$ in and around the deformable drop case. In this model, $R e=47.6, P e=793.73$ and $R_{\mu}=50$ (simulation \#6 in Table 2) 


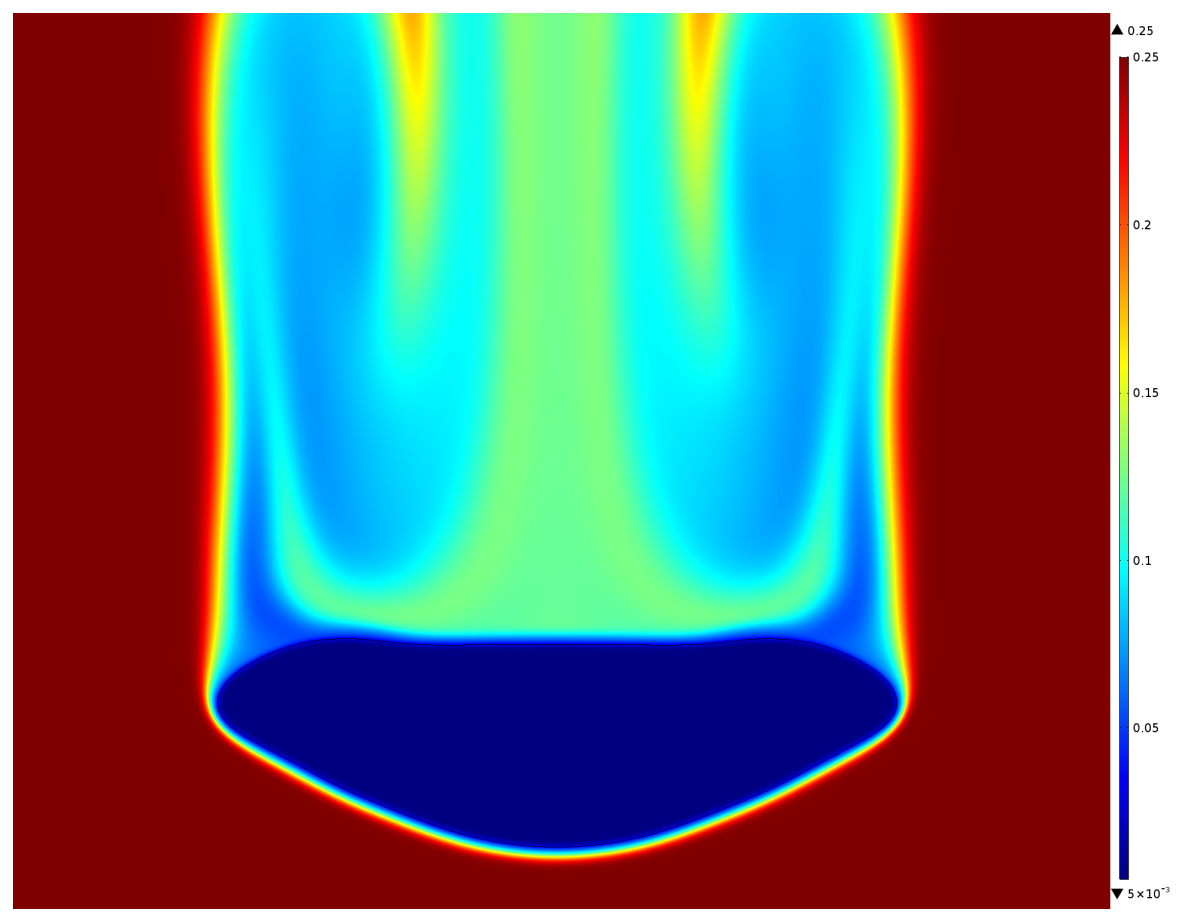

Figure 19: View of the viscosity field (in Pa.s) around the strongly deformable drop when a temperature dependent viscosity is considered. 

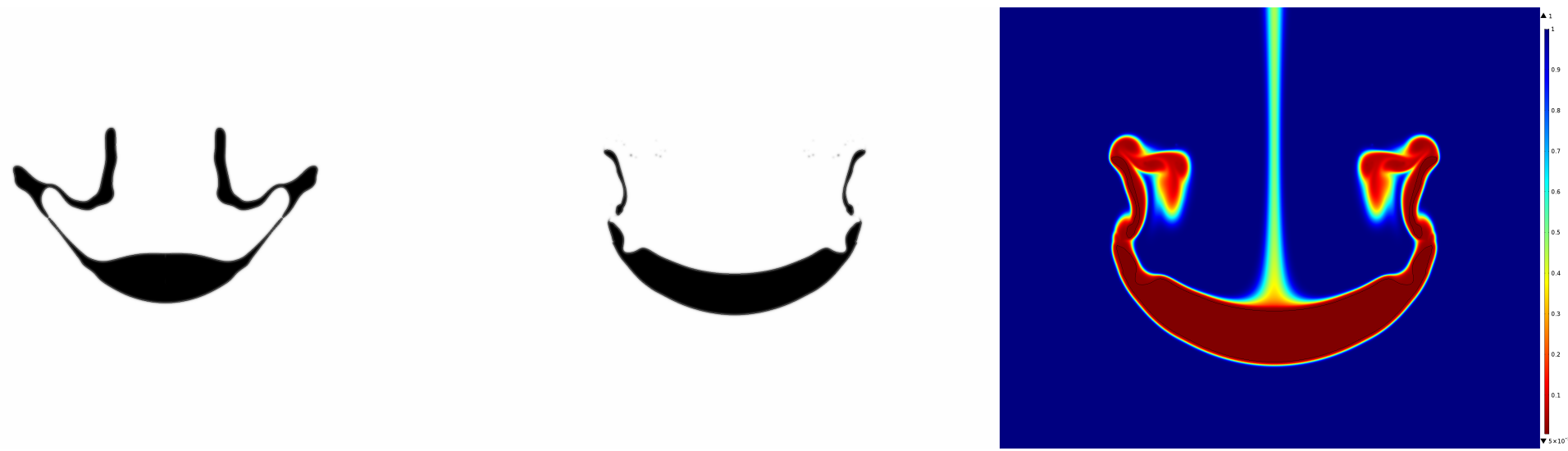

Figure 20: Final form at breaking of the drop for a constant viscosity magma ocean (Left) and a temperature dependent viscosity magma ocean (Middle). On the right, view of the viscosity field (in Pa.s) around the drop in the later case. In this model, $R e=50, P e=3330$ and $R_{\mu}=200$ (simulation \#10 in Table 2). 

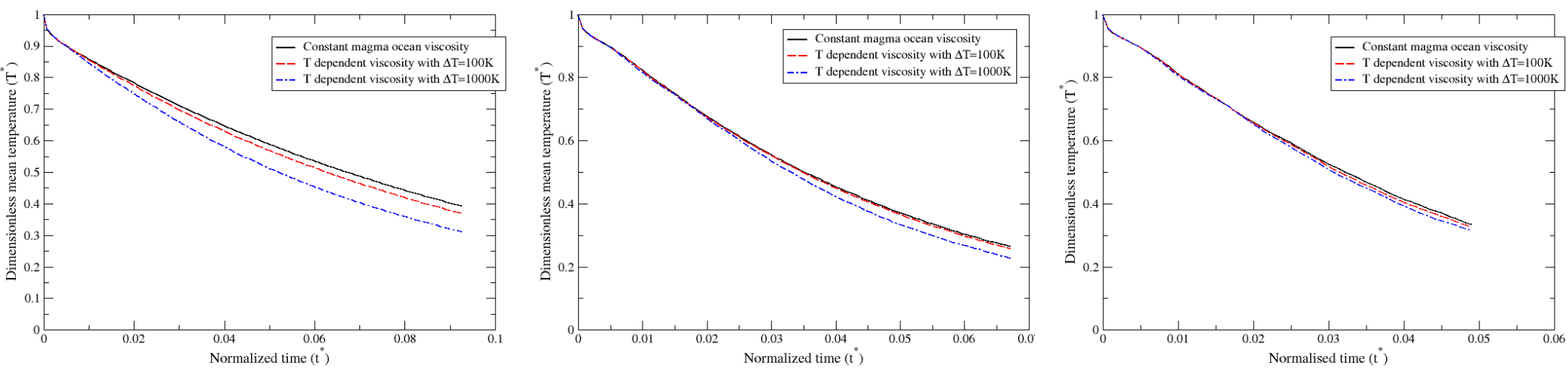

Figure 21: Comparison of the dimensionless temperature as a function of normalized time for a non-deformable drop (Left), a weakly deformable drop (Middle) and a strongly deformable drop (Right). The black lines present the constant magma ocean viscosity. The red (blue) dashed lines present the temperature dependent viscosity with $\Delta T=100 \mathrm{~K}(\Delta T=1000 \mathrm{~K})$. 

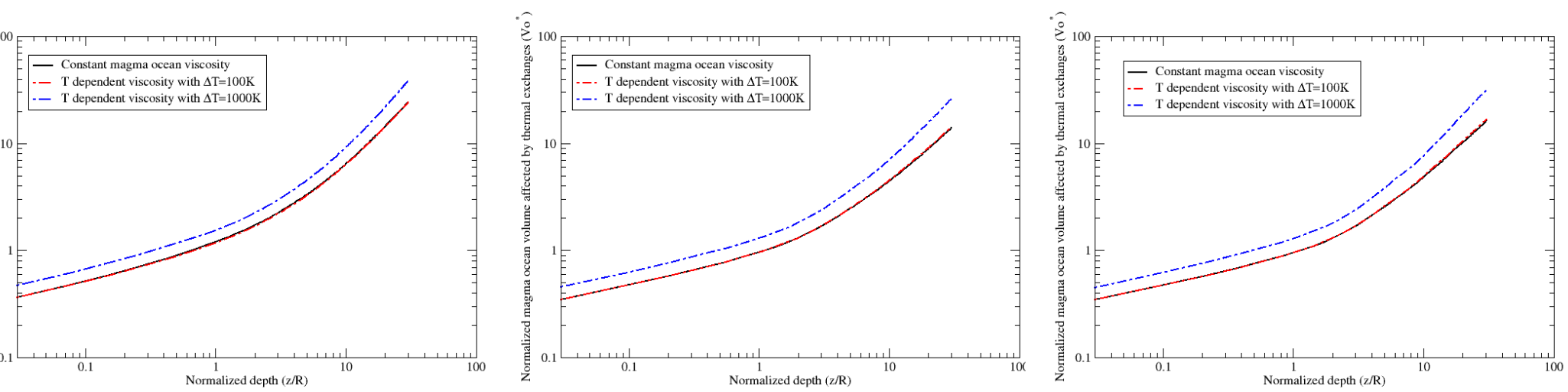

Figure 22: Comparison of normalized heated magma ocean volume as a function of normalized time for a non-deformable drop (Left), a weakly deformable drop (Middle) and a strongly deformable drop (Right). The black lines present the constant magma ocean viscosity. The red (blue) dashed lines present the temperature dependent viscosity with $\Delta T=100 \mathrm{~K}(\Delta T=$ $1000 \mathrm{~K})$. 